\title{
A Nonlocal Multiscale Fatigue Model
}

\author{
Jacob Fish and Caglar Oskay \\ Civil and Environmental Engineering Department \\ Rensselaer Polytechnic Institute, Troy, NY, 12180, USA
}

\begin{abstract}
A nonlocal multiscale model in time domain is developed for fatigue life predictions. The method is based on the mathematical homogenization theory with almost periodic fields. The almost periodicity reflects the effects of irreversible deformations in time domain in the form of accumulation of damage. Multiple temporal scales are introduced to decompose the original boundary value problem into micro-chronological (temporal unit cell) and macrochronological (homogenized) problems. A nonlocal Gurson type constitutive law is revisited for cyclic loading, calibrated and validated against fatigue crack propagation experiments on 316L austenitic stainless steel specimens.
\end{abstract}

Keywords: temporal homogenization, almost periodicity, fatigue life prediction, multiscale modeling, crack initiation, crack propagation.

\section{Introduction}

Fatigue of solids and structures is a multiscale phenomenon in space and time. The existence of multiple spatial scales is evident due to the presence of cracks and/or voids which may be orders of magnitude smaller than the structural component. Multiple temporal scales exist because of disparity between the period of loading and the overall structural fatigue life.

The primary phenomenological fatigue life prediction tool today is the so-called "total life" approach. By this approach, fatigue life is characterized by the stress-life (S-N) curves which relate the range of applied cyclic stresses to the number of load cycles to failure. When significant yielding is expected around a crack tip, the strain-life $(\varepsilon-\mathrm{N})$ curves are commonly used instead. The $\varepsilon-\mathrm{N}$ curves relate the range of applied cyclic strains (total or plastic) to the number of load cycles to failure. Such experimental characterizations are generally limited to small structural components or specimens. For larger assemblies, sole experimentation may not be feasible and far fields are typically computed numerically. The drawback of such a mixed experimental-computational approach is that it fails to account for force redistribution caused by damage accumulation.

One of the most widely used empirical fatigue life models is due to Paris and Erdogan [1]. The so-called Paris' law relates the crack growth rate to the range of stress intensity factors using a power representation. Paris' law, which was originally developed for the ideal condition of small scale yielding, was enhanced and generalized to incorporate various mechanisms such as R-effects, 
closure, threshold limits and others (e.g., see $[2,3,4,5])$. Unfortunately, the issues of modeling short cracks and the necessity for embedding initial macrocracks remain unresolved at large.

Direct Numerical Simulations (DNS) of fatigue crack growth by means of cycle-by-cycle simulations have been attempted in the context of the cohesive theory (e.g., Refs. [6, 7]). Obviously, DNS is not a feasible approach for simulating large scale systems subjected to high cycle fatigue. The so-called Cycle Jump Simulation (CJS) represents one of the first attempts to approximate the response of the direct cycle-by-cycle simulation $[8,9,10]$ using coarser time scales. The CJS has been found to perform reasonably well for relatively simple constitutive models such as those based on isotropic continuum damage theory. However, a mathematical framework based on the CJS approach that would satisfy governing equations in space and time for a general class of constitutive models remains an elusive task.

In this manuscript we present an alternative approach based on the mathematical homogenization theory in time domain with almost periodic fields. Almost periodicity is a byproduct of irreversible processes, such as damage accumulation, which violates the condition of temporal periodicity. We assume, however, that the non-periodic contribution is a perturbation of the periodic part. In addition, we assume that micro- and macro-chronological displacement fields are of the same order of magnitude. This is in contrast to the classical (spatial) mathematical homogenization where the microscale displacement field is taken as a perturbation of the macroscale field. Spatial homogenization in the presence of non-periodic conditions have been previously investigated using stochastic $[11,12,13,14]$ and deterministic $[15,16,17,18,19]$ methods. Mathematical analysis of the spatial homogenization theory with almost- and non-periodic fields have been conducted in Refs. [20, 21, 22, 23, 24].

The Gurson-Tvergaard-Needleman (GTN) [25] model serves as a basis for modeling of microvoid nucleation, growth, and coalescence processes, and subsequent propagation of macrocracks up to failure. Kinematic hardening and irreversible damage are employed to generate the hysteretic behavior, to prevent shakedown and premature crack arrest. It is well known that the degradation of material properties caused by progressive cavitation mechanism in the GTN model ultimately causes spurious mesh sensitivity of the response fields, rendering its predictions questionable. For monotonic loading, such mesh sensitive local models are commonly regularized using localization limiters including nonlocal gradient (e.g., [26]) and integral (e.g., [27]) type formulations, micro-polar continuum (e.g., [28]), and several others (e.g., [29, 30, 31]).

For cyclic loading, there are two scenarios that may give rise to mesh sensitivity: (i) loss of ellipticity at the progressive stages of damage growth, and (ii) unbounded damage growth rate caused by crack tip singularity. The latter has been investigated by Peerlings et al. [32] in the context of high cycle fatigue for quasi-brittle materials. They showed that for isotropic damage mechanics model, which preserves ellipticity of governing equations up to failure (damage parameter equals to one), mesh sensitivity is caused by condition ii. They concluded that a gradient type nonlocal regularization is effective as a localization limiter for high cycle fatigue of quasi-brittle materials. The GTN model considered in the present manuscript introduces additional challenges. In addition to the unbounded growth rate at the crack tip, the presence of plastic deformation may result in loss of ellipticity of the governing equations. To the best of the authors' knowledge, the present study is the first attempt to assess the performance of the nonlocal GTN model under the conditions of cyclic loading. In this manuscript, we focus on the development, calibration and validation of the nonlocal multiscale model of fatigue based on the integral version of the nonlocal GTN model, which is an extension of the 
local fatigue model developed in [33, 34].

The remaining of this manuscript is organized as follows. Section 2 reviews the fundamental aspects of the temporal homogenization in the presence of almost periodic fields. The nonlocal GTN model and the associated boundary value problem are presented in Section 3. Section 4 presents the formulation of the nonlocal multiscale model. Section 5 focuses on the computational aspects and the implementation details. The local and nonlocal multiscale models are compared in Section 6. In Section 7, the nonlocal multiscale fatigue model is first calibrated using fatigue experiments on 316L austenitic stainless steel specimens. Subsequently, the calibrated model is validated against a separate and independent set of fatigue crack propagation experiments. Conclusions and future research directions are discussed in Section 8.

\section{Multiple temporal scales and almost periodicity}

In a typical fatigue process, accumulation of damage, and the resulting macrocrack initiation and propagation is relatively slow, compared to rapid fluctuations of displacements within each load cycle. The disparity between the two characteristic time scales naturally introduces multiple time coordinates. In this study, we consider a two scale decomposition of time by defining a macrochronological scale denoted by the intrinsic time coordinate, $t$, and a micro-chronological scale denoted by the fast time coordinate, $\tau$. These two scales are related through a scaling parameter

$$
\tau=\frac{t}{\zeta} ; \quad 0<\zeta \ll 1
$$

in which, $\zeta\left(=\tau_{\mathrm{o}} / t_{\mathrm{r}}\right)$, is defined by the ratio of the characteristic lengths in micro- and macrochronological time, denoted by $\tau_{\mathrm{o}}$ and $t_{\mathrm{r}}$, respectively. The response fields, $\phi$, are assumed to depend on two temporal scales

$$
\phi^{\zeta}(\mathbf{x}, t)=\phi(\mathbf{x}, t, \tau(t))
$$

where, $\phi^{\zeta}$ is a rapidly oscillating function in time; and $\mathbf{x}$ denotes spatial coordinates. Time differentiation in the presence of multiple temporal scales is obtained using Eq. (1)

$$
\dot{\phi}^{\zeta}(\mathbf{x}, t)=\dot{\phi}(\mathbf{x}, t, \tau)=\phi_{, t}(\mathbf{x}, t, \tau)+\frac{1}{\zeta} \phi_{, \tau}(\mathbf{x}, t, \tau)
$$

in which, the comma followed by a subscript coordinate denotes partial derivative; and superposed dot is a total time derivative.

In this study, response fields are assumed to be almost periodic in time domain to account for the irreversible processes of damage accumulation. Almost periodicity implies that at the neighboring points in a spatial or temporal domain homologous by periodicity, the change in the response function is small but nonzero (in contrast to the case of local periodicity in which the change in response functions is zero). A typical almost periodic function is depicted in Figure 1.

The space of almost periodic functions, $\mathcal{T}$, is defined as:

$$
\mathcal{T}:=\{\phi \mid \phi(\mathbf{x}, t, \tau+k \kappa)-\phi(\mathbf{x}, t, \tau)=O(\zeta)\} \quad \forall k \in \mathbb{Z}
$$

A function $\phi \in \mathcal{T}$ is said to be $\kappa$-almost periodic if it belongs to the space of almost periodic functions. Attention is restricted on a subspace of $\mathcal{T}$ denoted by $\hat{\mathcal{T}}$

$$
\hat{\mathcal{T}}:=\left\{\phi_{a p} \mid \phi_{a p}(\mathbf{x}, t, \tau)=\phi_{p}(\mathbf{x}, t, \tau)+\zeta \tau \bar{\phi}(\mathbf{x})\right\}
$$


where, $\bar{\phi}$ is an arbitrary function constant in time; and $\phi_{p}$ is a $\kappa$-periodic function

$$
\phi_{p}(\mathbf{x}, t, \tau+k \kappa)=\phi_{p}(\mathbf{x}, t, \tau)
$$

Setting $\phi_{p}^{\zeta}(\mathbf{x}, t)=\phi_{p}(\mathbf{x}, t, t / \zeta)$, the well known weak convergence of the periodic fields is given as [35]:

$$
\lim _{\zeta \rightarrow 0} \int_{T} \phi_{p}^{\zeta}(\mathbf{x}, t) d t \rightarrow \int_{T}\left\langle\phi_{p}(\mathbf{x}, t, \tau)\right\rangle d t
$$

for any subset $T$ in $\mathbb{R}$. The periodic temporal homogenization $(\mathrm{PTH})$ operator, $\langle\cdot\rangle$, is given by:

$$
\langle\cdot\rangle:=\frac{1}{|\kappa|} \int_{\kappa}(\cdot) d \tau
$$

Defining $\phi_{a p}^{\zeta}(\mathbf{x}, t)=\phi_{a p}(\mathbf{x}, t, t / \zeta)$, it can be easily observed that Eq. (7) does not hold for almost periodic fields

$$
\begin{aligned}
\lim _{\zeta \rightarrow 0} \int_{T} \phi_{a p}^{\zeta} d t & =\lim _{\zeta \rightarrow 0} \int_{T} \phi_{p}^{\zeta} d t+\left.\bar{\phi} \frac{t^{2}}{2}\right|_{T} \\
\int_{T}\left\langle\phi_{a p}\right\rangle d t & =\int_{T}\left\langle\phi_{p}\right\rangle d t+\left.\left.\frac{\zeta}{2} \frac{\bar{\phi}}{|\kappa|} \tau^{2}\right|_{\kappa} t\right|_{T}
\end{aligned}
$$

The above serves as a motivation for an alternative definition of the averaging operator that would satisfy the weak convergence relation (Eq. (7)) for almost periodic functions in $\hat{\mathcal{T}}$. Such an almost periodic temporal homogenization (APTH) operator can be constructed in the rate form

$$
\mathfrak{M}\left(\phi_{a p}\right)_{, t}(\mathbf{x}, t):=\left\langle\dot{\phi}_{a p}\right\rangle(\mathbf{x}, t)
$$

so that the weak convergence relation (Eq. (7)) is satisfied for almost periodic functions in $\hat{\mathcal{T}}$

$$
\lim _{\zeta \rightarrow 0} \int_{T} \phi_{a p}^{\zeta}(\mathbf{x}, t) d t \rightarrow \int_{T} \mathfrak{M}\left(\phi_{a p}(\mathbf{x}, t, \tau)\right) d t
$$

Equation (11) may be verified by substituting the definition of the almost periodic functions given by Eq. (5) into the right hand side, and using (7)

$$
\begin{aligned}
\int_{T} \mathfrak{M}\left(\phi_{a p}\right) d t & =\int_{T}\left(\int_{0}^{t}\left[\frac{1}{\zeta}\left\langle\phi_{a p, \tau}\right\rangle+\left\langle\phi_{a p}\right\rangle_{, s}\right] d s\right) d t=\int_{T}\left(\int_{0}^{t}\left[\bar{\phi}+\left\langle\phi_{p}\right\rangle_{, s}\right] d s\right) d t \\
& =\int_{T}\left(t \bar{\phi}+\left\langle\phi_{p}\right\rangle\right) d t=\left.\bar{\phi} \frac{t^{2}}{2}\right|_{T}+\int_{T}\left\langle\phi_{p}\right\rangle d t
\end{aligned}
$$

The APTH and PTH operations are equivalent if applied to periodic fields.

Remark 1: : To further clarify the differences between the PTH and APTH operators, consider an illustrative example:

$$
\phi(t, \tau)=a_{0} \sin \left(\frac{2 \pi}{\zeta \tau_{0}} \tau\right)+\tau \bar{\phi}+a_{1}\left[1-\exp \left(-t / t_{d}\right)\right]
$$

where, the first term represents the rapidly oscillatory periodic function; the second reflects the non-periodic linear variation; and the last term depicts the slow macro-chronological variation. Figure 2 compares the functions $\mathfrak{M}\left(\phi_{a p}\right)$ and $\left\langle\phi_{a p}\right\rangle$ with the original function $\phi^{\zeta}$ as $\zeta \rightarrow 0$. Parameter values, $a_{0}=2.5, a_{1}=10.0, \tau_{0}=1$, and $\bar{\phi}=0.07$ were used in the illustration. The figure clearly shows that $\left\langle\phi_{a p}\right\rangle$ does not adequately tracks the average response of the almost periodic function, whereas $\mathfrak{M}\left(\phi_{a p}\right)$ does precisely that. 


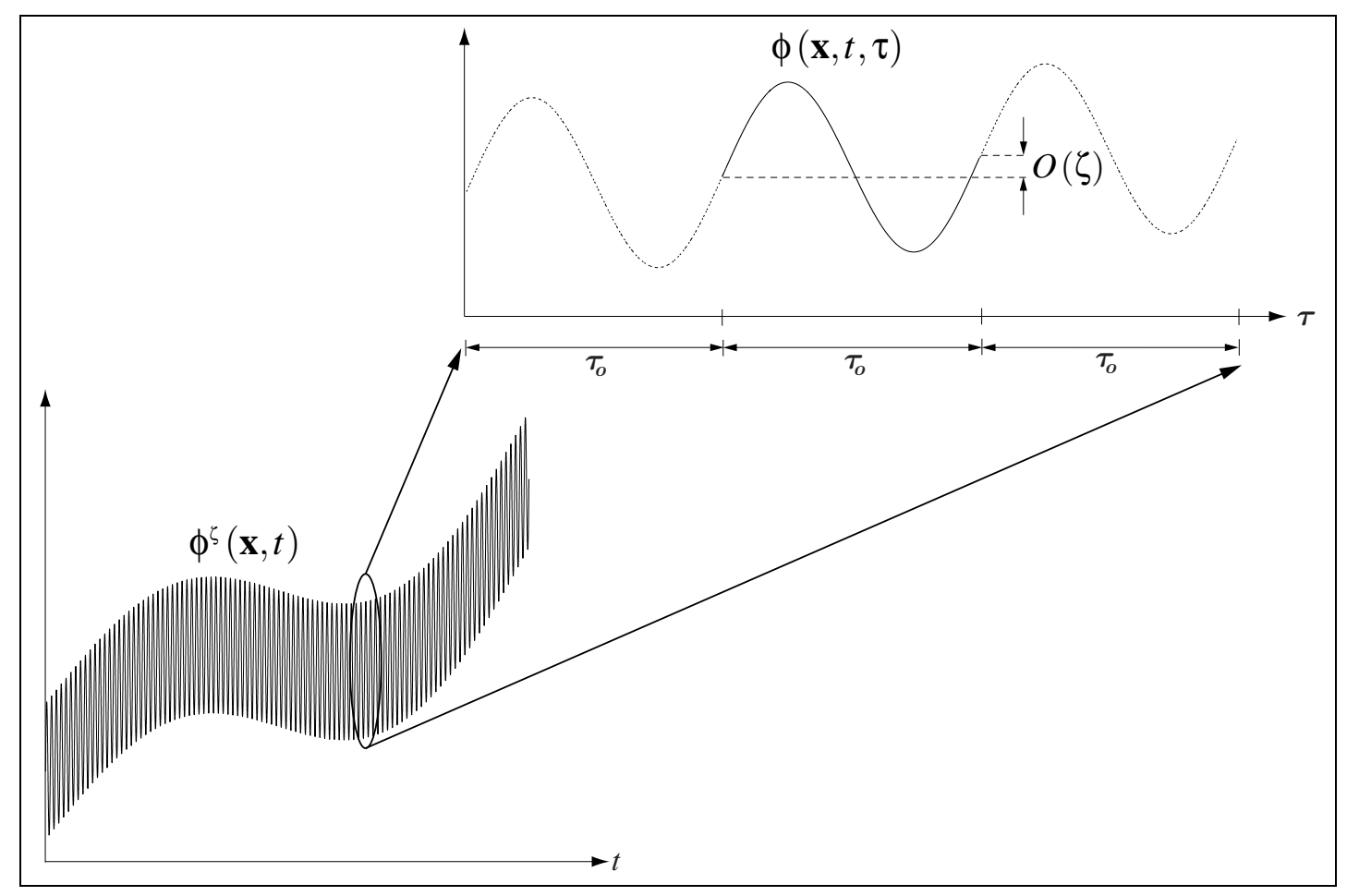

Figure 1: Almost periodic function.

\section{Nonlocal GTN model}

Progressive failure of ductile materials is commonly idealized using phenomenological elastoplastic constitutive models with a cumulative damage mechanism. One of the most widely known models of this type is due to Gurson [36]. The original Gurson's model is based on the limit analysis of a spherical unit cell with a spherical void inclusion at its center, and a Von-Mises type matrix material. A number of improvements to the original model have been proposed including the incorporation of void nucleation and coalescence mechanisms [37, 38], effects of non-spherical void inclusions [39], and others. The so-called Gurson-Tvergaard-Needleman (GTN) model, which accounts for the nucleation and coalescence effects, has been validated for monotonic [40] and cyclic [41] loading conditions.

From the numerical point of view, the GTN model is known to exhibit spurious mesh sensitivity when loading extends to the softening regime. Mesh sensitivity is characterized by localization of strains to the smallest possible volume admitted by the finite element mesh. We defer the discussion on the localization phenomenon and various localization limiters to the references discussed in the introduction of this manuscript. In the present study, we revisit the integral type of nonlocal formulation of the GTN model by defining a nonlocal consistency parameter which governs the evolution equations of hardening, void growth, and void nucleation.

We start by defining the boundary value problem 


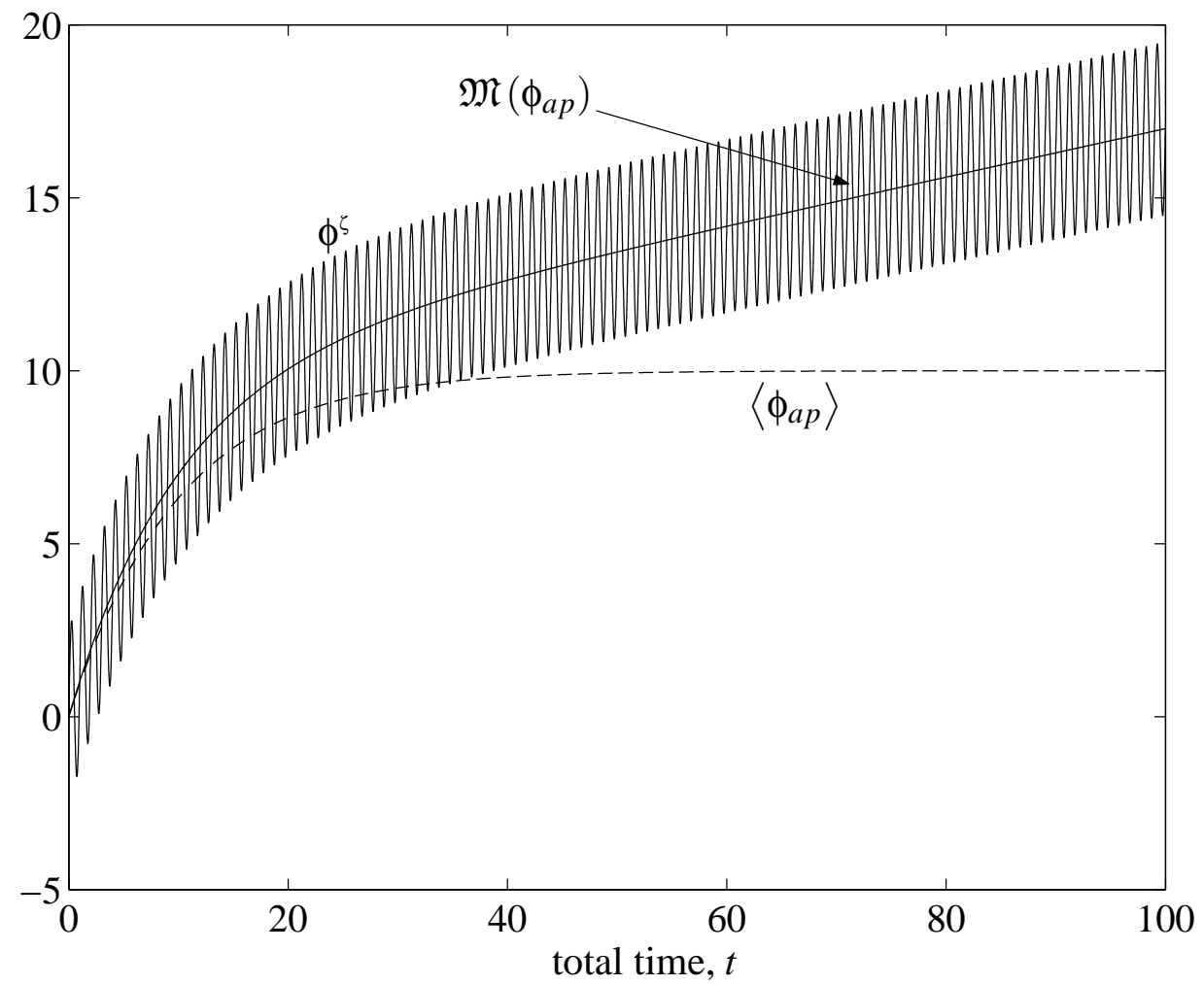

Figure 2: Decomposition of the response fields with respect to periodic and almost periodic temporal homogenization operators. 


$$
\begin{aligned}
\nabla \cdot \boldsymbol{\sigma}^{\zeta} & +\mathbf{b}^{\zeta}=\mathbf{0} & & \text { on } \Omega \times\left(0, t_{\mathrm{o}}\right) \\
\dot{\boldsymbol{\sigma}}^{\zeta} & =\mathbf{L}:\left(\dot{\boldsymbol{\varepsilon}}^{\zeta}-\dot{\boldsymbol{\mu}}^{\zeta}\right) & & \text { on } \Omega \times\left(0, t_{\mathrm{o}}\right) \\
\boldsymbol{\varepsilon}^{\zeta} & =\frac{1}{2}\left(\nabla \mathbf{u}^{\zeta}+\mathbf{u}^{\zeta} \nabla\right) & & \text { on } \Omega \times\left(0, t_{\mathrm{o}}\right) \\
\mathbf{u}^{\zeta} & =\mathbf{u}^{\prime} & & \text { on } \Omega \\
\mathbf{u}^{\zeta} & =\overline{\mathbf{u}}^{\zeta} & & \text { on } \Gamma_{u} \times\left(0, t_{\mathrm{o}}\right) \\
\mathbf{n} \boldsymbol{\sigma}^{\zeta} & =\mathbf{t}^{\zeta} & & \text { on } \Gamma_{t} \times\left(0, t_{\mathrm{o}}\right)
\end{aligned}
$$

where, $\mathbf{u}^{\zeta}$ is the displacement vector; $\boldsymbol{\sigma}^{\zeta}$ is the stress tensor; $\boldsymbol{\varepsilon}^{\zeta}$, and, $\boldsymbol{\mu}^{\zeta}$ are the total and plastic strain tensors, respectively; $\mathbf{L}$ is the tensor of elastic moduli; $\nabla$ is the vector differential operator given by $\nabla=\left(\partial / \partial x_{1}, \partial / \partial x_{2}, \partial / \partial x_{3}\right)$ in Cartesian coordinates; $(\cdot \nabla)=(\nabla \cdot)^{\mathbf{T}}$, superscript $\mathbf{T}$ indicates the transpose; $\mathbf{b}^{\zeta}$ is the body force; $\Omega$ and $\Gamma$ are the spatial problem domain and its boundary, respectively; $t_{\mathrm{o}}$ is the temporal problem domain; $\mathbf{u}^{\prime}$ is the initial displacement field; $\overline{\mathbf{u}}^{\zeta}$ and $\mathbf{t}^{\zeta}$ are the prescribed displacements and tractions on the boundaries $\Gamma_{u}$ and $\Gamma_{t}$, respectively, where $\Gamma=\Gamma_{u} \cup \Gamma_{t}$ and $\Gamma_{u} \cap \Gamma_{t}=\emptyset$. The analysis is restricted to small deformations.

The yield function of the GTN model is expressed as:

$$
\Phi^{\zeta}:=\Phi^{\zeta}\left(\boldsymbol{\sigma}^{\zeta}, \mathbf{H}^{\zeta}\right)=\frac{\left(q^{\zeta}\right)^{2}}{\left(\sigma_{F}^{\zeta}\right)^{2}}+2 \hat{f}^{\zeta} \cosh \left(-\frac{3}{2} \frac{p^{\zeta}}{\sigma_{F}^{\zeta}}\right)-1-\left(\hat{f}^{\zeta}\right)^{2}=0
$$

where, $\mathbf{H}$ denotes the set of internal state variables

$$
q^{\zeta}=\sqrt{\frac{3}{2} \mathbf{s}^{\zeta}: \mathbf{s}^{\zeta},} \quad \mathbf{s}^{\zeta}=\mathbf{B}^{\zeta}+p^{\zeta} \boldsymbol{\delta}, \quad \mathbf{B}^{\zeta}=\boldsymbol{\sigma}^{\zeta}-\boldsymbol{\alpha}^{\zeta}, \quad p^{\zeta}=-\frac{1}{3} \mathbf{B}^{\zeta}: \boldsymbol{\delta}
$$

$\boldsymbol{\alpha}^{\zeta}$ is the center of the yield surface; and $\boldsymbol{\delta}$ is the second order identity tensor. The radius of the yield surface, $\sigma_{F}^{\zeta}$, is defined as [42]:

$$
\sigma_{F}^{\zeta}=(1-b) \sigma_{y}+b \sigma_{M}^{\zeta}
$$

in which, $\sigma_{y}$ and $\sigma_{M}^{\zeta}$ are the initial yield stress and matrix flow stress, respectively; and $b \in[0,1]$ is a constant. $b=1, b=0$, and $b \in] 0,1[$ correspond to the pure isotropic, kinematic, and mixed hardening conditions, respectively.

The void coalescence function is commonly modeled using the piecewise linear function of the void volume fraction [38]

$$
\hat{f}^{\zeta}=\left\{\begin{array}{cc}
f^{\zeta} & f^{\zeta}<f_{c} \\
f_{c}+\left(\frac{1}{q_{1}}-f_{c}\right) \frac{f^{\zeta}-f_{c}}{f_{f}-f_{c}} & f^{\zeta} \geq f_{c}
\end{array}\right.
$$

in which, $f_{c}$ and $f_{f}$ are material parameters; and $q_{1}$ is the Tvergaard constant. When $f^{\zeta}$ approaches $f_{f}, \hat{f}^{\zeta} \rightarrow 1 / q_{1}$, and the material looses its stress carrying capacity.

The flow of plastic strains is chosen to follow the normality rule

$$
\dot{\boldsymbol{\mu}}^{\zeta}=\lambda^{\zeta} \frac{\partial \Phi^{\zeta}}{\partial \boldsymbol{\sigma}^{\zeta}} ; \quad \lambda^{\zeta} \geq 0
$$


in which, $\lambda^{\zeta}$ is the consistency parameter.

The nonlocal GTN model is formulated by defining the nonlocal consistency parameter, $\bar{\lambda}^{\zeta}$, using the well known integral equation (e.g., [43])

$$
\bar{\lambda}^{\zeta}(\mathbf{x}, t)=\frac{1}{W(\mathbf{x})} \int_{\Omega} w(\mathbf{x}, \mathbf{s}) \lambda^{\zeta}(\mathbf{s}, t) d s
$$

where,

$$
W(\mathbf{x})=\int_{\Omega} w(\mathbf{x}, \mathbf{s}) d s \quad \mathbf{x} \in \bar{\Omega}
$$

and, $w(\mathbf{x}, \mathbf{s})$ is an attenuation function. In this study, we use

$$
w(\mathbf{x}, \mathbf{s})=\frac{1}{\left[1+\left(\frac{\|\mathbf{x}-\mathbf{s}\|}{l_{c}}\right)^{c_{1}}\right]^{c_{2}}}
$$

in which, $l_{c}$ is the characteristic material length. $c_{1}$ and $c_{2}$ define the shape of the attenuation curve and they are set to 8 and 2, respectively, as suggested in Ref. [44]. The nonlocal evolution equations of the GTN model are defined based on the nonlocal consistency parameter.

$$
\dot{f}^{\zeta}=\dot{f}_{\mathrm{gr}}^{\zeta}+\dot{f}_{\text {nuc }}^{\zeta}
$$

The void volume fraction is allowed to grow only after the onset of plastic yielding, and under tensile loading conditions

$$
\dot{f}_{\mathrm{gr}}^{\zeta}=q_{1} \bar{\lambda}^{\zeta}\left(1-f^{\zeta}\right)\left\langle\frac{\partial \Phi^{\zeta}}{\partial \boldsymbol{\sigma}^{\zeta}}: \boldsymbol{\delta}\right\rangle_{+}
$$

where, $\langle\cdot\rangle_{+}=[(\cdot)+|\cdot|] / 2$ are MacCauley brackets. A strain controlled void nucleation mechanism is adopted [37]

$$
\dot{f}_{\text {nuc }}^{\zeta}=\mathcal{A}^{\zeta}\left(\rho^{\zeta}\right) \dot{\rho}^{\zeta}
$$

where, $\mathcal{A}^{\zeta}$ is taken to have a normal distribution

$$
\mathcal{A}^{\zeta}\left(\rho^{\zeta}\right)=q_{1} \frac{f_{N}}{s_{N} \sqrt{2 \pi}} \exp \left\{-\frac{1}{2}\left(\frac{\rho^{\zeta}-\varepsilon_{N}}{s_{N}}\right)^{2}\right\} \quad \text { if } \frac{\partial \Phi^{\zeta}}{\partial \boldsymbol{\sigma}^{\zeta}}: \boldsymbol{\delta}>0 ; \quad \mathcal{A}^{\zeta}=0 \text { otherwise }
$$

in which, $\rho^{\zeta}$ is the equivalent plastic strain; $f_{N}$, is the volume fraction of void nucleating particles; $\varepsilon_{N}$ is the mean strain for nucleation; and $s_{N}$ is the standard deviation of nucleation.

The evolution of the effective plastic strain is given by:

$$
\dot{\rho}^{\zeta}=\frac{\bar{\lambda}^{\zeta}}{\left(1-f^{\zeta}\right) \sigma_{F}^{\zeta}} \mathbf{B}^{\zeta}: \frac{\partial \Phi^{\zeta}}{\partial \boldsymbol{\sigma}^{\zeta}}
$$

The equivalent plastic strain rate is related to the matrix flow strength

$$
\dot{\sigma}_{M}^{\zeta}=\left(\frac{E E_{t}}{E-E_{t}}\right) \dot{\rho}^{\zeta}=E^{t} \dot{\rho}^{\zeta}
$$

in which $E$ is the Young's modulus; and $E_{t}$ is the tangent to the uniaxial stress-strain diagram at a given stress level.

The evolution equation for the center of the yield surface is:

$$
\dot{\boldsymbol{\alpha}}^{\zeta}=\bar{\lambda}^{\zeta} Q^{\zeta} \mathbf{B}^{\zeta} ; \quad Q^{\zeta} \geq 0
$$

The value of $Q^{\zeta}$ is determined using the consistency condition. 


\section{Multiple temporal scale analysis}

We define the following decomposition of the almost periodic fields

$$
\phi(\mathbf{x}, t, \tau)=\mathfrak{M}(\phi)(\mathbf{x}, t)+\tilde{\phi}(\mathbf{x}, t, \tau)
$$

in which, $\phi$ denotes the displacement, strain, stress or internal state variable fields; $\mathfrak{M}(\phi)$ and $\tilde{\phi}$ correspond to the macro-chronological (homogenized) and the micro-chronological (oscillatory) parts of the response fields, respectively. Employing the definition of the APTH operator, the following relation is obtained

$$
\langle\tilde{\phi}\rangle_{, t}=-\frac{1}{\zeta}\left\langle\tilde{\phi}_{, \tau}\right\rangle
$$

In view of Eq. (35), we seek to decompose the boundary value problem defined in Section 3 to a set of coupled micro- and macro-chronological problems. Applying the APTH operator to Eq. (14), the macro-chronological equilibrium equations may be obtained

$$
\nabla \cdot \mathfrak{M}(\boldsymbol{\sigma})+\mathfrak{M}(\mathbf{b})=\mathbf{0} \quad \text { on } \Omega \times\left(0, t_{\mathrm{o}}\right)
$$

Decomposing the stresses and the body forces using Eq. (35) and subtracting Eq. (37) from the resulting equation yields the equilibrium equations for the micro-chronological problem. At a macro-chronological time instant, $t$, we have a unit cell problem in time domain

$$
\nabla \cdot \tilde{\boldsymbol{\sigma}}+\mathbf{b}-\mathfrak{M}(\mathbf{b})=\mathbf{0} \quad \text { on } \Omega \times\left(0, \tau_{\mathrm{o}}\right)
$$

The original constitutive relation may be decomposed by applying Eq. (35) to the stress, strain, and plastic strain tensors, substituting the resulting terms into Eq. (15), and gathering the terms of equal order

$$
\begin{aligned}
O\left(\zeta^{-1}\right): & \tilde{\boldsymbol{\sigma}}_{, \tau} & =\mathbf{L}:\left(\tilde{\boldsymbol{\varepsilon}}_{, \tau}-\tilde{\boldsymbol{\mu}}_{, \tau}\right) \quad \text { on } \Omega \times\left(0, \tau_{\mathrm{o}}\right) \\
O(1): & \mathfrak{M}(\boldsymbol{\sigma})_{, t}+\tilde{\boldsymbol{\sigma}}_{, t} & =\mathbf{L}:\left(\mathfrak{M}(\boldsymbol{\varepsilon})_{, t}-\mathfrak{M}(\boldsymbol{\mu})_{, t}+\tilde{\boldsymbol{\varepsilon}}_{, t}-\tilde{\boldsymbol{\mu}}_{, t}\right)
\end{aligned}
$$

The micro-chronological constitutive relation is given by Eq. (39a). Applying the PTH operator to Eq. (39) and using Eq. (36), the constitutive equation for the macro-chronological problem is obtained

$$
\mathfrak{M}(\boldsymbol{\sigma})_{, t}=\mathbf{L}:\left(\mathfrak{M}(\boldsymbol{\varepsilon})_{, t}-\mathfrak{M}(\boldsymbol{\mu})_{, t}\right) \quad \text { on } \Omega \times\left(0, t_{\mathrm{o}}\right)
$$

The kinematic equation may be decomposed into a micro- and macro-chronological parts by applying Eq. (35) to the displacement field, substituting the resulting terms into Eq. (16), and exploiting the linearity of the APTH operator

$$
\begin{aligned}
\tilde{\boldsymbol{\varepsilon}} & =\frac{1}{2}(\nabla \tilde{\mathbf{u}}+\tilde{\mathbf{u}} \nabla) \\
\mathfrak{M}(\boldsymbol{\varepsilon}) & =\frac{1}{2}(\nabla \mathfrak{M}(\mathbf{u})+\mathfrak{M}(\mathbf{u}) \nabla)
\end{aligned}
$$

The flow rules of the micro- and macro-chronological problems are formulated by considering a two-scale decomposition of the local consistency parameter. At a material point, $\mathbf{x}$, we have

$$
\lambda(t, \tau)=\frac{1}{\zeta} \lambda^{1}(t, \tau)+\lambda^{\mathrm{o}}(t)
$$


where, $\lambda^{1}$ and $\lambda^{0}$ may be interpreted as the consistency parameters induced by the micro- and macro-chronological loadings, respectively. Applying Eq. (35) to the plastic strain tensor, substituting the resulting terms and the above decomposition of the consistency parameter to the flow rule (Eq. (24)), and gathering equal order terms yield:

$$
\begin{aligned}
O\left(\zeta^{-1}\right): & \tilde{\boldsymbol{\mu}}_{, \tau} & =\lambda^{1} \frac{\partial \Phi}{\partial \boldsymbol{\sigma}} \\
O(1): & \mathfrak{M}(\boldsymbol{\mu})_{, t}+\tilde{\boldsymbol{\mu}}_{, t} & =\lambda^{0} \frac{\partial \Phi}{\partial \boldsymbol{\sigma}}
\end{aligned}
$$

The flow rule for the micro-chronological problem is given by Eq. (44a). Applying the PTH operator to Eqs. (44), and using Eq. (36) gives:

$$
\mathfrak{M}(\boldsymbol{\mu})_{, t}=\lambda^{o}\left\langle\frac{\partial \Phi}{\partial \boldsymbol{\sigma}}\right\rangle+\frac{1}{\zeta}\left\langle\tilde{\boldsymbol{\mu}}_{, \tau}\right\rangle
$$

where, the yield condition is expressed in terms of the micro- and macro-chronological fields using the function relation

$$
\Phi(\boldsymbol{\sigma}, \mathbf{H})=\Phi(\mathfrak{M}(\boldsymbol{\sigma}), \tilde{\boldsymbol{\sigma}}, \mathfrak{M}(\mathbf{H}), \tilde{\mathbf{H}})
$$

The evaluation of the micro-chronological plastic strains for the nonlocal GTN model is detailed in Appendix A. The macro-chronological plastic strains, stresses, total strains and internal state variables are evaluated using a two-step algorithm described in Section 5.

The evolution equations of the internal state variables (Eqs. (22), (29), (30), (32)-(34)) may be expressed in a general functional form as:

$$
\dot{\mathbf{H}}=\overline{\mathbf{h}}(\boldsymbol{\sigma}, \bar{\lambda}, \mathbf{H})
$$

or in terms of the macro- and micro-chronological fields

$$
\dot{\mathbf{H}}=\mathbf{h}\left(\mathfrak{M}(\boldsymbol{\sigma}), \tilde{\boldsymbol{\sigma}}, \bar{\lambda}^{o}, \bar{\lambda}^{1}, \mathfrak{M}(\mathbf{H}), \tilde{\mathbf{H}}\right)
$$

where,

$$
\bar{\lambda}^{i}(\mathbf{x}, t)=\frac{1}{W(\mathbf{x})} \int_{\Omega} w(\mathbf{x}, \mathbf{s}) \lambda^{i}(\mathbf{s}, t) d \mathbf{s}, \quad i=0,1
$$

The evolution equations may be decomposed as:

$$
\mathbf{h}=\frac{1}{\zeta} \mathbf{h}^{1}+\mathbf{h}^{\mathrm{o}}
$$

Decomposing the internal state variable fields based on Eq. (35) and using Eq. (3) yields:

$$
\begin{aligned}
\tilde{\mathbf{H}}_{, \tau} & =\mathbf{h}^{1}\left(\mathfrak{M}(\boldsymbol{\sigma}), \tilde{\boldsymbol{\sigma}}, \bar{\lambda}^{1}, \mathfrak{M}(\mathbf{H}), \tilde{\mathbf{H}}\right) \\
\mathfrak{M}(\mathbf{H})_{, t}+\tilde{\mathbf{H}}_{, t} & =\mathbf{h}^{\mathrm{o}}\left(\mathfrak{M}(\boldsymbol{\sigma}), \tilde{\boldsymbol{\sigma}}, \bar{\lambda}^{\mathrm{o}}, \mathfrak{M}(\mathbf{H}), \tilde{\mathbf{H}}\right)
\end{aligned}
$$

The micro-chronological evolution equation for the internal state variables is given by Eq. (51a). Applying the PTH operator to Eqs. (51) and using Eq. (36) gives the evolution equations of the macro-chronological problem

$$
\mathfrak{M}(\mathbf{H})_{, t}=\left\langle\mathbf{h}^{\mathrm{o}}\right\rangle+\frac{1}{\zeta}\left\langle\tilde{\mathbf{H}}_{, \tau}\right\rangle
$$


The evolution equations for the nonlocal GTN model are presented in Appendix B.

Substituting the displacement and stress decompositions into Eqs. (17)-(19), and applying the APTH operator yields the initial and the boundary conditions for the macro-chronological problem

$$
\begin{aligned}
\mathfrak{M}(\mathbf{u})(\mathbf{x}, t=0) & =\mathbf{u}^{\prime}(\mathbf{x}) & & \text { on } \Omega \\
\mathfrak{M}(\mathbf{u}) & =\mathfrak{M}(\overline{\mathbf{u}}(\mathbf{x}, t, \tau)) & & \text { on } \Gamma_{u} \times\left(0, t_{\mathrm{o}}\right) \\
\mathbf{n} \mathfrak{M}(\boldsymbol{\sigma}) & =\mathfrak{M}(\mathbf{t}(\mathbf{x}, t, \tau)) & & \text { on } \Gamma_{t} \times\left(0, t_{\mathrm{o}}\right)
\end{aligned}
$$

Similarly, the micro-chronological initial and boundary conditions are obtained by applying Eq. (35) to the displacement and stress fields, substituting the resulting equation into Eqs. (17)(19) and subtracting Eqs. (53)-(55) from the resulting equations

$$
\begin{aligned}
\tilde{\mathbf{u}}(\mathbf{x}, t, \tau=0) & =\mathbf{0} & & \text { on } \Omega \\
\tilde{\mathbf{u}}(\mathbf{x}, t, \tau) & =\overline{\mathbf{u}}-\mathfrak{M}(\overline{\mathbf{u}}) & & \text { on } \Gamma_{u} \times\left(0, \tau_{\mathrm{o}}\right) \\
\mathbf{n} \tilde{\boldsymbol{\sigma}}(\mathbf{x}, t, \tau) & =\mathbf{t}-\mathfrak{M}(\mathbf{t}) & & \text { on } \Gamma_{t} \times\left(0, \tau_{\mathrm{o}}\right)
\end{aligned}
$$

The coupled macro-chronological and the micro-chronological problems are summarized in Box 1.

\section{Numerical aspects}

\subsection{Nonlocal consistency parameter}

The introduction of the nonlocal consistency parameter leads to the integro-differential equation for the local consistency parameter

$$
\lambda(\mathbf{x}, t)=\frac{\frac{\partial \phi}{\partial \boldsymbol{\sigma}}: \mathbf{L}: \dot{\boldsymbol{\varepsilon}}}{\frac{\partial \phi}{\partial \boldsymbol{\sigma}}: \mathbf{L}: \frac{\partial \phi}{\partial \boldsymbol{\sigma}}}-\frac{H}{\frac{\partial \phi}{\partial \boldsymbol{\sigma}}: \mathbf{L}: \frac{\partial \phi}{\partial \boldsymbol{\sigma}}} \frac{1}{W(\mathbf{x})} \int_{\Omega} w(\mathbf{x}, \mathbf{s}) \lambda(\mathbf{s}, t) d \mathbf{s}
$$

The expression for $H$ and the details of the derivation of the above equation are presented in Appendix A. Solution of the boundary value problem with such nonlocal characterization of internal state variables requires somewhat cumbersome integration techniques [45]. To reduce the computational complexity we use the following approximation of the nonlocal consistency parameter

$$
t_{n+1} \bar{\lambda}\left(\boldsymbol{x}_{i}\right)=\frac{g_{i}}{W_{i}} t_{n+1} \lambda\left(\boldsymbol{x}_{i}\right)+\frac{1}{W_{i}} \sum_{\substack{j=1 \\ j \neq i}}^{n_{g p}} g_{j} w\left(\boldsymbol{x}_{i}, \boldsymbol{x}_{j}\right)_{t_{n}} \lambda\left(\boldsymbol{x}_{j}\right)
$$

where, $W_{i}=W\left(\boldsymbol{x}_{i}\right) ; \boldsymbol{x}_{i}$ denotes the spatial coordinates of the $i$ th integration point; $n_{g p}$ is the total number of integration points in the mesh; $g_{i}$ are the weight factors for the numerical integration; and the left subscript denote temporal discretization. Discretizing Eq. (59) and substituting into Eq. (60) yields

$$
t_{n+1} \lambda\left(\boldsymbol{x}_{i}\right)=\frac{\frac{\partial \phi}{\partial \boldsymbol{\sigma}}: \mathbf{L}:\left.\dot{\boldsymbol{\varepsilon}}\right|_{t_{n+1}}}{\frac{g_{i}}{W_{i}} H+\frac{\partial \phi}{\partial \boldsymbol{\sigma}}: \mathbf{L}:\left.\frac{\partial \phi}{\partial \boldsymbol{\sigma}}\right|_{t_{n+1}}}+\frac{H / W_{i}}{\frac{g_{i}}{W_{i}} H+\frac{\partial \phi}{\partial \boldsymbol{\sigma}}: \mathbf{L}:\left.\frac{\partial \phi}{\partial \boldsymbol{\sigma}}\right|_{t_{n+1}}} \sum_{\substack{j=1 \\ j \neq i}}^{n_{g p}} g_{j} w\left(\boldsymbol{x}_{i}, \boldsymbol{x}_{j}\right)_{t_{n}} \lambda\left(\boldsymbol{x}_{j}\right)
$$




\section{Macro-chronological problem:}

$\begin{array}{lll}\text { Equilibrium equation: } & \nabla \cdot \mathfrak{M}(\boldsymbol{\sigma})+\mathfrak{M}(\mathbf{b})=\mathbf{0} & \text { on } \Omega \times\left(0, t_{\mathrm{o}}\right)\end{array}$

Constitutive equation:

$$
\mathfrak{M}(\boldsymbol{\sigma})_{, t}=\mathbf{L}:\left(\mathfrak{M}(\boldsymbol{\varepsilon})_{, t}-\mathfrak{M}(\boldsymbol{\mu})_{, t}\right) \text { on } \Omega \times\left(0, t_{\mathrm{o}}\right)
$$

Kinematic equation:

$$
\mathfrak{M}(\boldsymbol{\varepsilon})=\frac{1}{2}(\nabla \mathfrak{M}(\mathbf{u})+\mathfrak{M}(\mathbf{u}) \nabla) \quad \text { on } \Omega \times\left(0, t_{0}\right)
$$

Initial condition:

$$
\mathfrak{M}(\mathbf{u})(\mathbf{x}, t=0)=\mathbf{u}^{\prime}(\mathbf{x})
$$

on $\Omega$

Boundary conditions:

$$
\begin{aligned}
\mathfrak{M}(\mathbf{u}) & =\mathfrak{M}(\overline{\mathbf{u}}(\mathbf{x}, t, \tau)) \\
\mathbf{n M}(\boldsymbol{\sigma}) & =\mathfrak{M}(\mathbf{t}(\mathbf{x}, t, \tau)) \\
\mathfrak{M}(\boldsymbol{\mu})_{, t} & =\lambda^{\mathrm{o}}\left\langle\frac{\partial \Phi}{\partial \boldsymbol{\sigma}}\right\rangle+\frac{1}{\zeta}\left\langle\tilde{\boldsymbol{\mu}}_{, \tau}\right\rangle
\end{aligned}
$$$$
\text { on } \Gamma_{u} \times\left(0, t_{\mathrm{o}}\right)
$$$$
\text { on } \Gamma_{t} \times\left(0, t_{\mathrm{o}}\right)
$$

Flow rule:

Evolution equations:

$$
\mathfrak{M}(\mathbf{H})_{, t}=\left\langle\mathbf{h}^{\mathrm{o}}\right\rangle+\frac{1}{\zeta}\left\langle\tilde{\mathbf{H}}_{, \tau}\right\rangle
$$

\section{Micro-chronological problem:}

Equilibrium equation:

Constitutive equation:

Kinematic equation:

Initial condition:

Boundary conditions:

Flow rule:

Evolution equations:

$$
\begin{aligned}
\nabla \cdot \tilde{\boldsymbol{\sigma}}+\mathbf{b}-\mathfrak{M}(\mathbf{b}) & =\mathbf{0} & & \text { on } \Omega \times\left(0, \tau_{\mathrm{o}}\right) \\
\tilde{\boldsymbol{\sigma}}_{, \tau} & =\mathbf{L}:\left(\tilde{\boldsymbol{\varepsilon}}_{, \tau}-\tilde{\boldsymbol{\mu}}_{, \tau}\right) & & \text { on } \Omega \times\left(0, \tau_{\mathrm{o}}\right) \\
\tilde{\boldsymbol{\varepsilon}} & =\frac{1}{2}(\nabla \tilde{\mathbf{u}}+\tilde{\mathbf{u}} \nabla) & & \text { on } \Omega \times\left(0, \tau_{\mathrm{o}}\right) \\
\tilde{\mathbf{u}}(\mathbf{x}, t, \tau=0) & =\mathbf{0} & & \text { on } \Omega \\
\tilde{\mathbf{u}}(\mathbf{x}, t, \tau) & =\overline{\mathbf{u}}-\mathfrak{M}(\overline{\mathbf{u}}) & & \text { on } \Gamma_{u} \times\left(0, \tau_{\mathrm{o}}\right) \\
\mathbf{n} \tilde{\boldsymbol{\sigma}}(\mathbf{x}, t, \tau) & =\mathbf{t}-\mathfrak{M}(\mathbf{t}) & & \text { on } \Gamma_{t} \times\left(0, \tau_{\mathrm{o}}\right)
\end{aligned}
$$$$
\tilde{\mu}_{, \tau}=\lambda^{1} \frac{\partial \Phi}{\partial \boldsymbol{\sigma}}
$$$$
\tilde{\mathbf{H}}_{, \tau}=\mathbf{h}^{1}\left(\mathfrak{M}(\boldsymbol{\sigma}), \tilde{\boldsymbol{\sigma}}, \bar{\lambda}^{1}, \mathfrak{M}(\mathbf{H}), \tilde{\mathbf{H}}\right)
$$

Box 1: Governing equations of the macro- and micro-chronological problems. 
It can be observed that the local consistency parameter, ${ }_{t_{n+1}} \lambda$, may be evaluated locally using Eq. (61). Similar methods have been previously employed for the nonlocal characterization of other variants of the GTN model (e.g., [44]).

\subsection{Adaptive multi-scale methodology}

The micro- and macro-chronological boundary value problems formulated in the previous section and summarized in Box 1 are coupled through the constitutive relationships. In this study, we adopted a staggered solution algorithm with adaptive macro-chronological time stepping control. The proposed algorithm is convenient in the sense that it facilitates the use of commercially available finite element packages solely by invoking user supplied material subroutines.

The proposed adaptive algorithm for evaluating the micro- and macro-chronological problems is summarized in Box 2. The objective of the algorithm is to evaluate the micro- and macrochronological response fields denoted by $\tilde{\phi}(\mathbf{x}, t, \tau)$ and $\mathfrak{M}(\phi)(\mathbf{x}, t)$, respectively, at time $t=t_{n+1}$, given the response fields at time $t=t_{n}\left(t_{n}<t_{n+1}\right)$. The solution at macro-chronological time $t_{n+1}$ is computed adaptively. From a qualitative viewpoint, the macro-chronological step size, $\Delta t$ $\left(=t_{n+1}-t_{n}\right)$ is chosen to be relatively large if the computed response is smooth, otherwise, the step size is reduced to maintain accuracy. To this extent, a vector of control variables, $\boldsymbol{\omega}(\boldsymbol{\sigma}, \boldsymbol{\mu}, \mathbf{H})$, is defined as a function of the response fields. Given a converged state at $t=t_{n}$, the control variable vector at $t=t_{n+1}$ is computed twice at each integration point; first using a single macro-chronological time step, $\Delta t$ (to compute ${\left(t_{n+1} ; \Delta t\right)} \omega)$, then using two successive steps with macro-chronological step size $\Delta t / 2$ (to compute ${\left(t_{n+1} ; \Delta t / 2\right)}^{\omega})$. The error at $t=t_{n+1}$ is then given by

$$
\mathbf{E}^{\boldsymbol{\omega}}=\left.\right|_{\left.{ }_{t_{n+1} ; \Delta t}\right)} \boldsymbol{\omega}-{ }_{\left.t_{n+1} ; \Delta t / 2\right)} \boldsymbol{\omega} \mid \leq \mathbf{E}^{t o l}
$$

in which, $E^{t o l}$ is the vector of predefined error tolerances for the control variables; and the left subscript $(\cdot ; \cdot)$ denotes the current time and the time step size used in the stress update. If the computed error exceeds the error tolerance vector, $\mathbf{E}^{\text {tol }}$, at any integration point, the macro-chronological time step size is reduced based on the value of the errors and the procedure is repeated until the error tolerances are met. The micro-chronological time step size $\delta \tau=\tau_{k+1}-\tau_{k}$ is controlled by the accuracy of the stress update procedure.

Remark 2: In this study, the vector of the control variables includes the void volume fraction, $f$, and the Euclidian norm of the plastic strain deviator, $\mathbf{s}_{\mu}$

$$
\boldsymbol{\omega}=\left\{f,\left\|\mathbf{s}_{\boldsymbol{\mu}}\right\|_{2}\right\}^{\mathrm{T}} ; \quad \mathbf{s}_{\boldsymbol{\mu}}=\boldsymbol{\mu}-\frac{1}{3} \boldsymbol{\delta}: \boldsymbol{\mu}
$$

For a given macro-chronological time step size, $\Delta t$, the integration of the macro-chronological fields is conducted based on a two-step procedure. This procedure ensures the equilibrium equations and the consistency condition to be satisfied at every macro-chronological step. Figure 3 illustrates the basic structure of the proposed two-step procedure. The micro-chronological loading induced macro-chronological fields are introduced to the converged state of the macrochronological fields at $t=t_{n}$, to obtain an intermediate configuration, ${ }_{t_{n+1}} \mathfrak{M}(\phi)^{*}$ (step 5 of Box 2). The macro-chronological loading is subsequently applied to the intermediate configuration to evaluate the current state of the macro-chronological fields, ${ }_{t_{n+1}} \mathfrak{M}(\phi)$. The stress updates of the micro- 
and macro-chronological problems are carried out using a return mapping algorithm based on the method first proposed in [46] for isotropic hardening, and further extended to account for kinematic hardening in [47].

The aforementioned adaptive algorithm requires data transfer between the micro- and macrochronological problems at each time step. In this study, the information between the micro- and macro-chronological problems is communicated through an external batch processing to facilitate the use of a commercial finite element software. The ABAQUS analysis engine incorporating user supplied subroutines (UMAT) was employed to conduct the numerical simulations. The response information of the macro- and micro-chronological problems was stored on a hard disk.

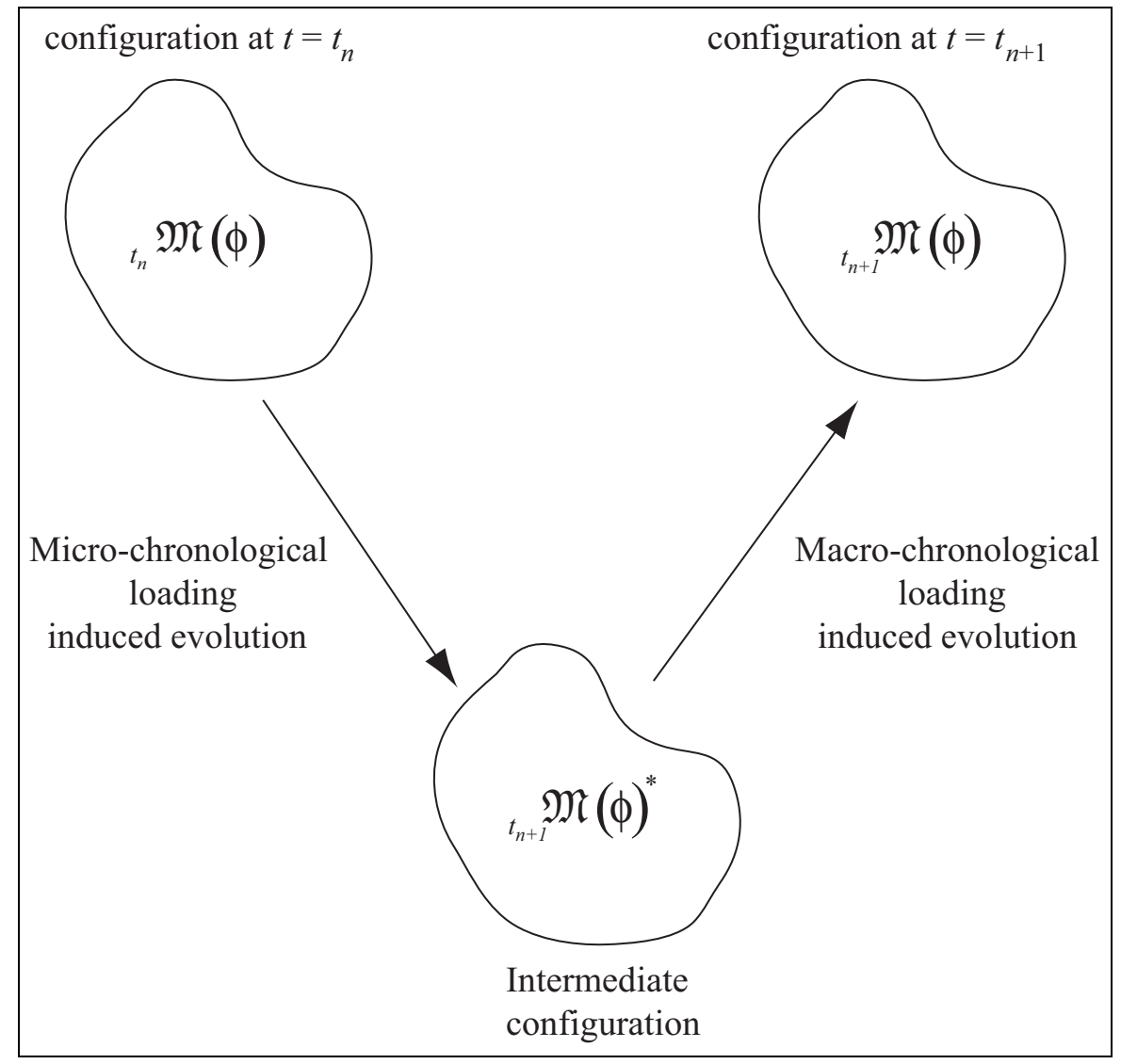

Figure 3: Schematic of the proposed two step update procedure.

\section{Mesh sensitivity studies}

The localization characteristics of the nonlocal multiscale model are assessed using fatigue crack propagation simulations in a rectangular panel with a blunted notch. Figure 4 displays the geometry of the panel and the applied vertical displacements. Plane strain conditions were assumed and a quarter of the panel was modeled due to symmetry. Three finite element meshes were considered with increased refinement along the path of the crack. Far fields were taken to be identical for all meshes. The path of the crack propagation was discretized uniformly with an average element size of $h=0.1,0.05$, and 0.025 units, and a small mesh transition zone is placed between the far fields 
Input: ${ }_{t_{n}} \mathfrak{M}(\phi)(\mathbf{x})$ and ${ }_{t_{n}} \tilde{\phi}(\mathbf{x}, \tau)$

Output: $\Delta t,_{t_{n+1}} \mathfrak{M}(\phi)$ and ${ }_{t_{n+1}} \tilde{\phi}(\tau)$.

1. Initialize: $I D_{\text {tol }} \leftarrow$ false, $E_{F} \leftarrow 1$

2. Compute $t_{n} \boldsymbol{\omega} \equiv \boldsymbol{\omega}\left({ }_{t_{n}} \boldsymbol{\sigma}, t_{n} \mathbf{H}, t_{n} \boldsymbol{\mu}\right)$ and ${ }_{t_{n}} \delta \boldsymbol{\omega}$ at each integration point

$$
{ }_{t_{n}} \delta \boldsymbol{\omega}\left(\mathbf{x}_{k}\right)={ }_{t_{n}} \boldsymbol{\omega}\left(\mathbf{x}_{k}, \tau_{\mathrm{o}}\right){ }_{{ }_{t_{n}}} \boldsymbol{\omega}\left(\mathbf{x}_{k}, 0\right) ; \quad \forall k \in 1,2, \ldots, n_{i p}
$$

$\left(* n_{i p}\right.$ : number of integration points $\left.*\right)$

3. Compute initial estimate of $\Delta t$ :

$$
\Delta t=\min _{j=1}^{n_{\omega d}}\left(\delta \omega_{j}^{a l l} / \max _{k=1}^{n_{i p}}\left[\delta \omega_{j}\left(\mathbf{x}_{k}, t\right)\right]\right) \tau_{\mathrm{o}}
$$

$\left(* n_{\omega d}:\right.$ number of dimensions of the control variable vector. $\left.*\right)$

$\left(* \delta \boldsymbol{\omega}^{\text {all }}\right.$ : maximum allowable accumulation within a load cycle. $\left.*\right)$

4. $\quad$ while $I D_{t o l}=$ false

5. $\quad$ Set $\Delta t \leftarrow \Delta t / E_{F}$ :

$$
{ }_{\left(t_{n+1} ; \Delta t\right.} \mathfrak{M}(\phi)^{*}={ }_{t_{n}} \mathfrak{M}(\phi)+\frac{\Delta t}{\tau_{\mathrm{o}}}\left[{ }_{t_{n}} \tilde{\phi}\left(\mathbf{x}, \tau_{\mathrm{o}}\right)-{ }_{t_{n}} \tilde{\phi}(\mathbf{x}, 0)\right]
$$

6. Apply the macro-chronological external force increments and solve for ${ }_{\left(t_{n+1} ; \Delta t\right)} \mathfrak{M}(\phi)$, using ${ }_{\left(t_{n+1} ; \Delta t\right)} \mathfrak{M}(\phi)^{*}$ as the initial values of a standard update procedure.

7. $\quad$ Solve micro-chronological IBVP for ${ }_{\left(t_{n+1} ; \Delta t\right)} \tilde{\phi}$ using ${ }_{\left(t_{n+1} ; \Delta t\right)} \mathfrak{M}(\phi)$

8. Set $\Delta t \leftarrow \Delta t / 2$, evaluate steps (5)-(7) in two increments to compute ${ }_{\left(t_{n+1} ; \Delta t / 2\right)} \mathfrak{M}(\phi)$ and $\left(t_{n+1} ; \Delta t / 2\right) \tilde{\phi}$

9. $\quad$ Compute ${ }_{\left(t_{n+1} ; \Delta t\right)} \boldsymbol{\omega}$ using ${\left(t_{n+1} ; \Delta t\right)} \mathfrak{M}(\phi)$ and ${ }_{\left(t_{n+1} ; \Delta t\right)} \tilde{\phi}$

10. Compute ${ }_{\left(t_{n+1} ; \Delta t / 2\right)} \boldsymbol{\omega}$ using ${ }_{\left(t_{n+1} ; \Delta t / 2\right)} \mathfrak{M}(\phi)$ and ${ }_{\left(t_{n+1} ; \Delta t / 2\right)} \tilde{\phi}$.

11. Evaluate ${\left(t_{n+1} ; \Delta t\right)} \delta \boldsymbol{\omega}$ and ${ }_{\left(t_{n+1} ; \Delta t / 2\right)} \delta \boldsymbol{\omega}$ at each integration point.

12.

$$
\begin{gathered}
E_{j}^{\boldsymbol{\omega}}=\max _{k=1}^{n_{i p}}\left(\left\|_{\left(t_{n+1} ; \Delta t\right)} \omega_{j}\left(\mathbf{x}_{k}, \tau\right)-{ }_{\left(t_{n+1} ; \Delta t / 2\right)} \omega_{j}\left(\mathbf{x}_{k}, \tau\right)\right\|_{W}\right) ; \quad \forall j \in 1,2, \ldots, n_{\omega d} \\
\|\cdot\|_{W}=\left(\int_{0}^{\tau_{0}}(\cdot)^{W} d \tau\right)^{1 / W} ; \quad \text { if } 1<W<\infty, \quad\|\cdot\|_{W}=\max (\cdot)\left(\mathbf{x}_{k}, \tau\right) ; \quad \text { if } W=\infty
\end{gathered}
$$

13. $\quad$ if $E_{j}^{\boldsymbol{\omega}} \leq E_{j}^{t o l} ; \forall j \in 1,2, \ldots, n_{\omega d}$ then $I D_{t o l} \leftarrow$ true; return

14. else

$$
E_{F}=\min \left(\max _{j=1}^{n_{\omega d}}\left[E_{j}^{\boldsymbol{\omega}} / E_{j}^{t o l}\right], E_{L}\right)
$$

\section{return}

$\left(* E_{L}\right.$ : maximum allowable time step size reduction factor. $\left.*\right)$

15. $t_{n+1} \leftarrow t_{n}+\Delta t$

Box 2: Adaptive algorithm. 
and the crack propagation path. Figure 5 shows the discretization at the tip of the blunted notch for each mesh.

The propagation of a fatigue crack is modeled by effectively eliminating (a small fraction of the residual stiffness is retained for stability) the elements for which the void volume fraction reaches $90 \%$ of the critical void volume fraction. Since the void volume fraction growth in GTN model is controlled by the plastic process, the issue of spurious damage growth and increase in damage zone well beyond the size of the characteristic material length (see [48]) was not observed. Reduced integration with hourglass control is employed to avoid partially vanishing elements.

Mechanical properties of the panel and the GTN model parameters are summarized in Table 1. The characteristic length, $l_{c}$, is chosen to be 0.13 which is slightly larger than the element size of the coarsest mesh. The effects of far fields on the nonlocal weight function is negligible and was ignored by setting $w(\mathbf{x}, \mathbf{s})$ to zero for $\|\mathbf{x}-\mathbf{s}\| \geq 1.5 l_{c}$.

The void coalescence mechanism is considered using a third order void coalescence function

$$
\hat{f}^{\zeta}=f^{\zeta}+\frac{6}{f_{f}^{3}}\left(\frac{1}{q_{1}}-f_{f}\right)\left[\left(\frac{f_{f}}{2}-f_{c}\right)+\left(\frac{f_{c}}{f_{f}}-\frac{1}{3}\right) f^{\zeta}\right]\left(f^{\zeta}\right)^{2}
$$

The constants of the third order void coalescence function were chosen to provide the best curve fit of a piecewise linear function of Eq. (23), and to satisfy the boundary conditions. Similar to the piecewise linear function, material looses its stress carrying capacity as void volume fraction approaches the critical value $\left(\hat{f}^{\zeta} \rightarrow 1 / q_{1}\right.$ as $\left.f \rightarrow f_{f}\right)$.

The uniaxial stress-strain curve of the matrix material was modeled using a piecewise power law

$$
\varepsilon=\left\{\begin{array}{cc}
\frac{\sigma}{E} & \sigma \leq \sigma_{Y} \\
\frac{\sigma_{Y}}{E}\left(\frac{\sigma}{\sigma_{Y}}\right)^{n} & \sigma>\sigma_{Y}
\end{array}\right.
$$

where, $n$ is the strain hardening exponent. The multiscale simulations were performed using the algorithmic parameters, $E_{L}=4, \delta \boldsymbol{\omega}^{\text {all }}=\{0.01,0.01\}$, and with error tolerances of $\mathbf{E}^{\text {tol }}=$ $\{0.0015,0.0015\}$.

Figure 6 displays the path of the fatigue crack simulated using the local GTN model as obtained after 500 load cycles. The damage zone clearly localizes to smaller regions and the rate of crack propagation increases as the mesh is refined. The fatigue crack path obtained under identical loading and topological conditions but with the nonlocal GTN model is shown in Fig. 7. It can be seen that the results obtained after 500 and 750 cycles are insensitive to the mesh size except for the coarsest mesh. The damage zone (i.e., the region with high void volume fraction) localizes to a finite region with a size comparable to the characteristic material length. The discrepancy between the results of the coarsest mesh and the finer meshes is attributed to the fact that the size of the elements in front of the crack tip is comparable to the characteristic material length.

\section{Calibration and validation of the GTN model}

Verification studies of the local multiscale fatigue model against cycle-by-cycle simulations were conducted in [33] for brittle materials and in [34] for ductile materials. In the present manuscript attention is restricted to calibration and experimental validation of the nonlocal multiscale model 
Table 1: Material properties of the panel with a blunted notch.

\begin{tabular}{|cccc|}
\hline & Poisson's ratio & Hardening parameter & Initial void volume fraction \\
$E / \sigma_{Y}=300$ & $v=0.3$ & $b=0.0$ & $f_{0}=0.0053$ \\
\hline Tvergaard constant, & Coalescence, & Nucleation, & Hardening exponent \\
$q_{1}=1.5$ & $f_{c}=0.15$ & $f_{N}=0.04$ & $n=10$ \\
& $f_{f}=0.25$ & $s_{N}=0.1$ & \\
& & $\varepsilon_{N}=0.3$ & \\
\hline
\end{tabular}

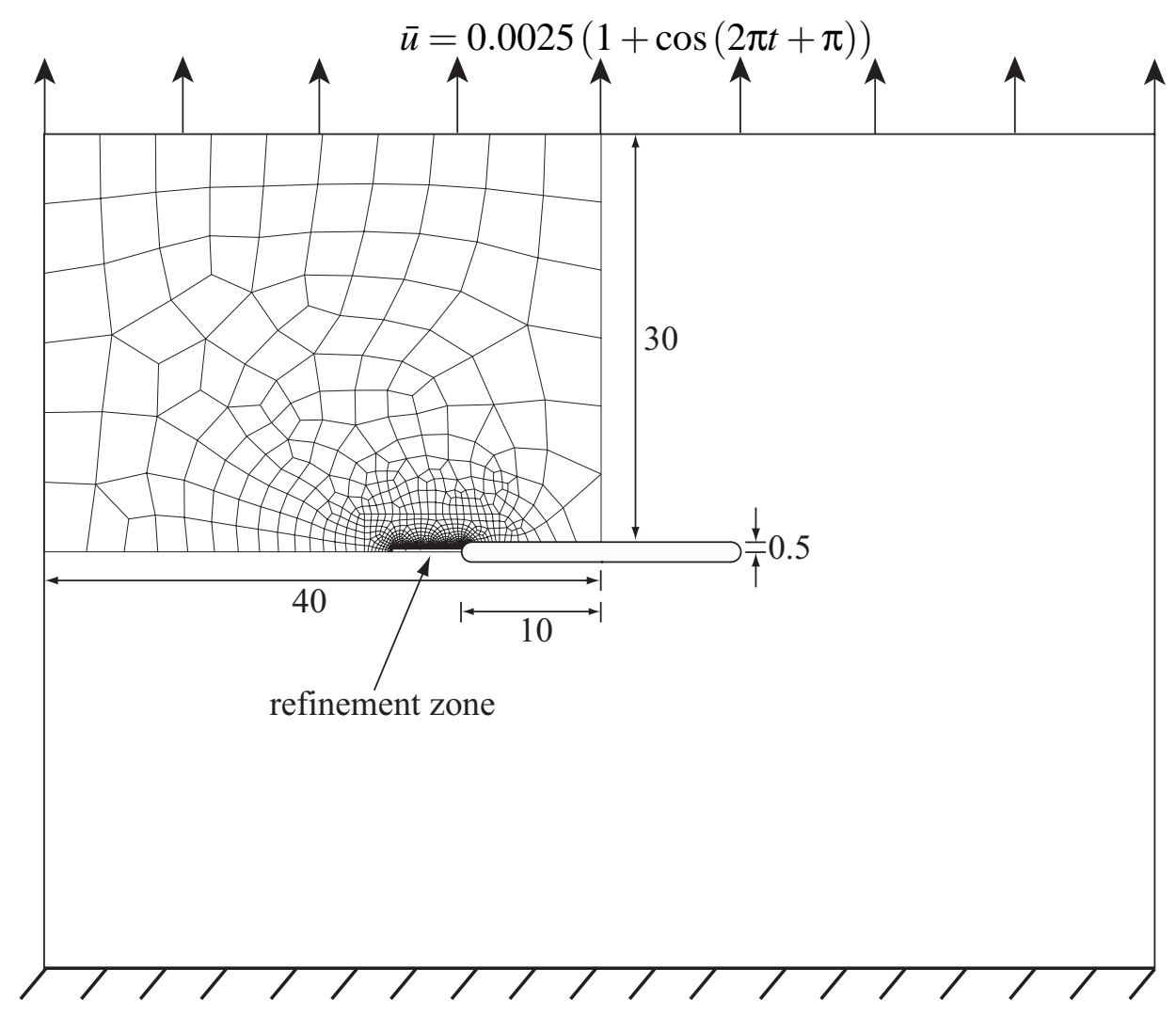

Figure 4: Geometry and the far field mesh of the panel with a blunted notch. 


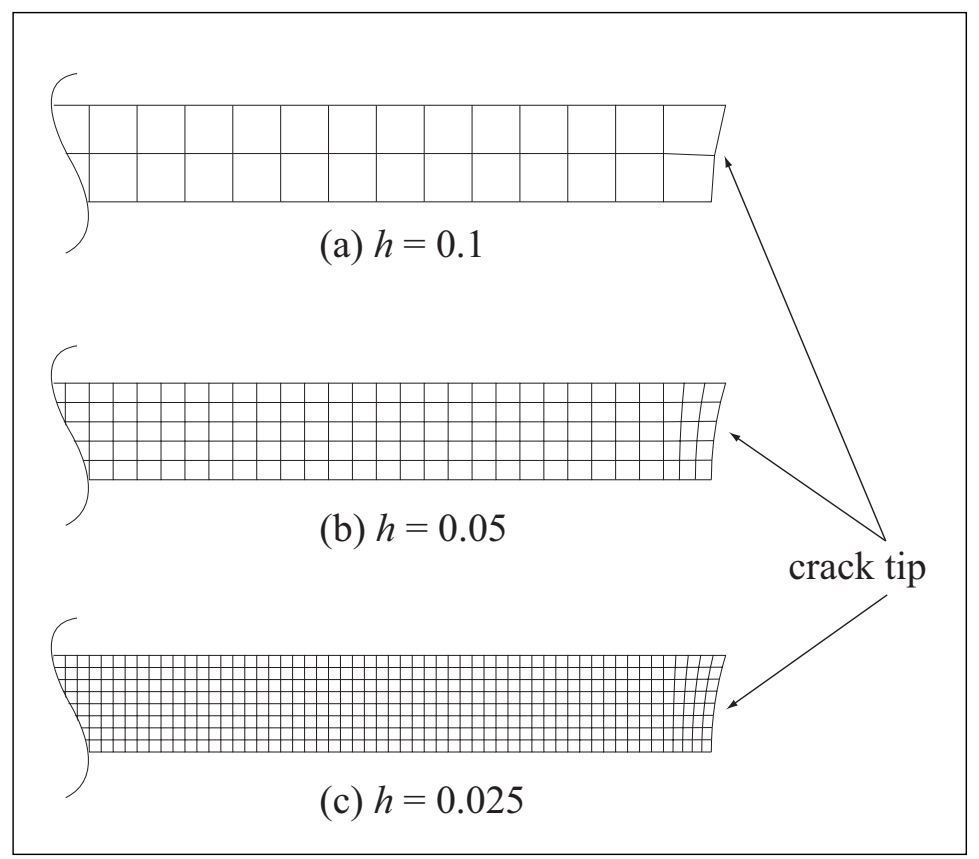

Figure 5: Crack tip discretizations used in the fatigue simulations of the panel with a blunted notch.

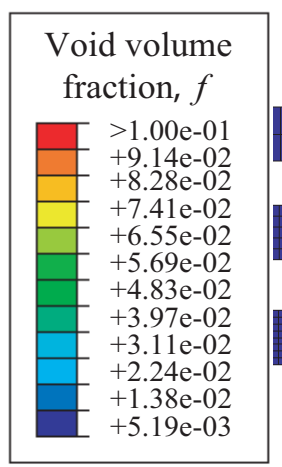

Cycle \# 500

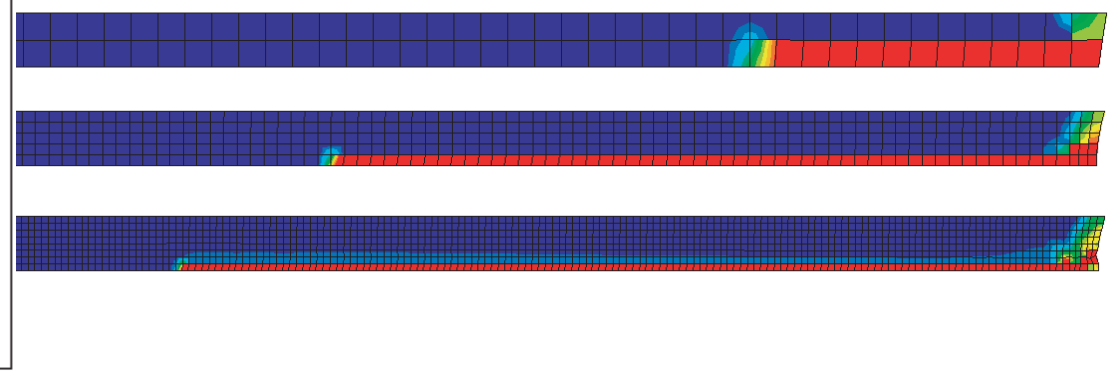

Figure 6: Local simulations. 
Cycle \# 500

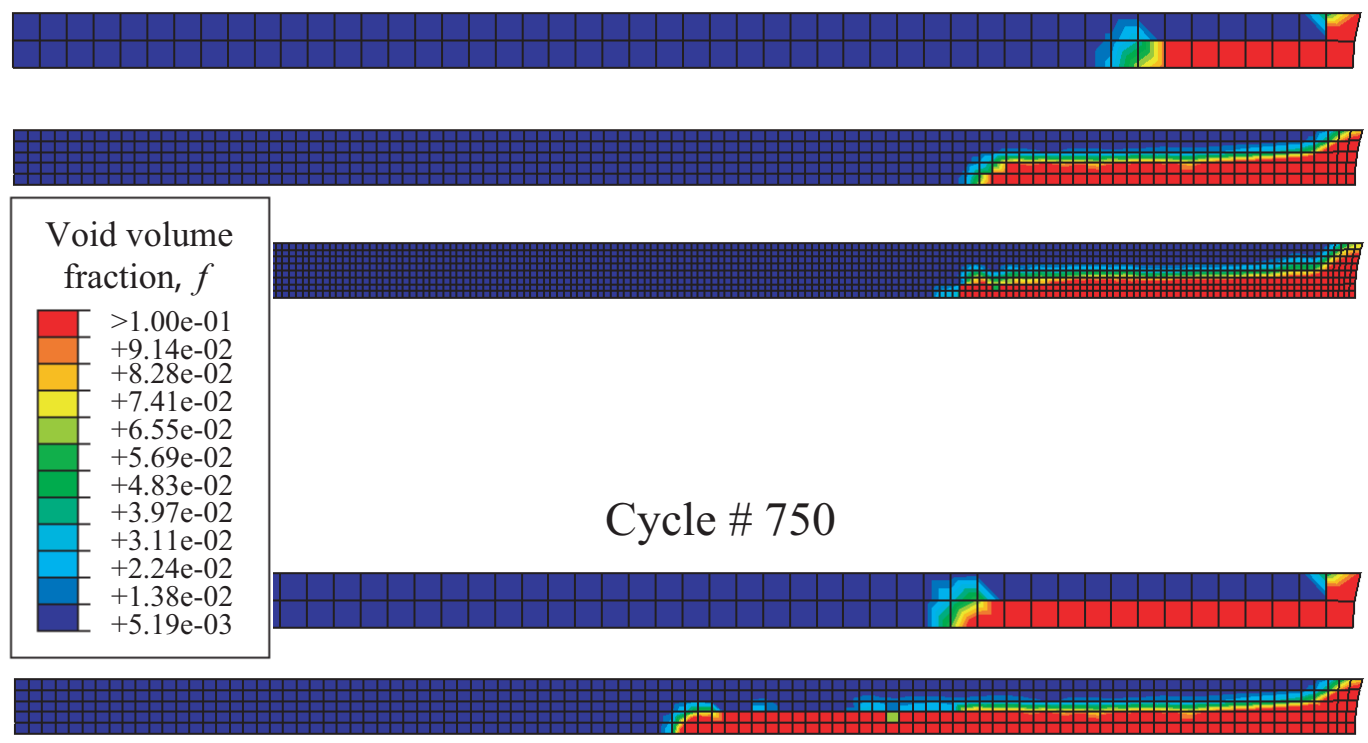

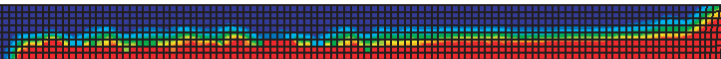

Figure 7: Nonlocal simulations.

for ductile metals. We consider two independent sets of fatigue experiments conducted on $316 \mathrm{~L}$ austenitic stainless steel specimens with similar chemical compositions. The first set is used for the calibration of parameters of the GTN model. The calibrated parameters were subsequently used to predict the propagation characteristics of a single macrocrack, and the results were compared to the observations of the second set of experiments. The model calibration was conducted using direct cycle-by-cycle simulations. After appropriate parameter values of the model were determined, the validation simulations were conducted using the nonlocal multiscale approach.

\subsection{Model calibration}

The fatigue response of the 316L stainless steel was previously investigated experimentally by Shi and Pluvinage [49] under isothermal and thermomechanical loading conditions. Cylindrical test samples were taken from a plate which has been solution treated and subsequently water quenched. Figure 8 displays the geometry and dimensions of the cylindrical fatigue specimens. The specimens were subjected to displacement controlled mechanical cycles of various magnitudes using a servo-hydraulic test machine. The initial and stabilized cyclic total stress and plastic strain ranges were recorded.

The GTN model is calibrated against the results of two isothermal fatigue experiments reported in Ref. [49]. The void volume fraction of the virgin material, $f_{0}$, was computed a-priori based on the chemical composition of the material using Franklin's empirical formula $\left(f_{0}=0.054(\% \mathrm{~S}-0.001 \% \mathrm{Mn})\right)$. Using the $\mathrm{S}$ and Mn contents of the 316 L stainless steel specimens given as 0.01 and 1.8, respectively, the initial void volume fraction is computed to be $f_{0}=0.022 \%$. The $0.2 \%$ proof stress of the material was obtained from the values suggested by Ref. [50] as $173 \mathrm{MPa}$. The nonlocal char- 
acteristic length was chosen to be of the order of the grain size $\left(l_{c}=80 \mu \mathrm{m}\right)$ as suggested in Ref. [32]. The void nucleation and coalescence mechanisms were excluded by setting $\mathcal{A}=0$, and $\hat{f}=f$.

Calibration of the remaining constitutive parameters which are the mixed hardening parameter, $b$, the Tvergaard constant, $q_{1}$, and the hardening curve of the GTN model is conducted using the simulations of two isothermal fatigue experiments under prescribed cyclic displacements with maximum amplitudes of $0.012 \mathrm{~mm}$ (specimen CLB1) and $0.016 \mathrm{~mm}$ (specimen CLB2) which corresponds to $1.2 \%$ and $1.6 \%$ engineering strains, respectively. The loading was applied symmetrically in the compressive-tensile direction with a triangular waveform. Simulations are conducted under the assumption of axisymmetric conditions and half of the cross-section is discretized due to symmetry as shown in Fig. 8. A small imperfection was introduced around the circumference of the specimen. The imperfection is a semi-circular region in the plane of the cross-section $(0.3 \mathrm{~mm}$ radius) with void concentrations linearly increasing toward the surface of the specimen $\left(f_{0}=0.1 \%\right.$ in elements adjacent to the surface).

The hardening curve, the mixed hardening parameter and the Tvergaard constant were evaluated by minimizing the discrepancy between the simulated and experimental values of the initial and stabilized stresses and plastic strains, and the overall fatigue lives of the specimens in a least squares sense. The Tvergaard constant, the mixed hardening parameter, and the strain hardening exponents were identified to be $0.22,1.1$, and 3.7 , respectively.

Figure 9 illustrates the evolution of the stress ranges $\left(\Delta \sigma=\left|\sigma_{\min }\right|+\left|\sigma_{\max }\right|\right)$ computed using the numerical simulations for specimens CLB1 and CLB2. The corresponding fatigue lives were predicted to be 500 and 330 cycles for specimens CLB1 and CLB2, respectively. The observed experimental fatigue lives of the two specimens were given as 550 and 295 cycles, respectively. The observed values for the stabilized stress ranges are given as 686 and 720 for CLB1 and CLB2, respectively, which are in reasonable agreement with the stress ranges predicted by the GTN model. The plastic strain ranges for the specimens CLB1 and CLB2 as predicted by the numerical simulations, and the observed experimental values are plotted in Fig. 10. Similarly, the plastic strains were predicted to be in the range of the experimental observations (within 8\%). The discrepancies between the experimental observations and the results of the numerical simulations are attributed to the limitation of the hardening laws used in the simulations (i.e., mixed kinematic-isotropic hardening). For low cycle fatigue, more elaborate hardening laws and, possibly, bounding surface and multi-yield surface approaches need to be investigated.

\subsection{Model validation}

The calibrated GTN model is then employed to predict the growth of fatigue cracks on specimens made of 316L stainless steel. Wheatley et al. [51] previously conducted experiments to investigate the propagation characteristics of cracks under fatigue loading and in the presence of overloads. They tested compact-tension (CT) specimens made of 316 stainless steel with chemical compositions similar to that of the cylindrical specimens used in the calibration of the GTN model. The CT specimens were $6 \mathrm{~mm}$ thick and $40 \mathrm{~mm}$ wide, and the initial notches were $12 \mathrm{~mm}$ long parallel to the bar length. Prior to the experiments, a $6 \mathrm{~mm}$ fatigue pre-crack was produced by high amplitude cyclic loading. Figure 11 illustrates the geometry of the CT specimens used in the experiments. A sinusoidal waveform with maximum amplitude of $3 \mathrm{kN}$ and an R-ratio of 0.1 was applied in the tensile direction. In the overload experiment, a single cycle overload with maximum amplitude 


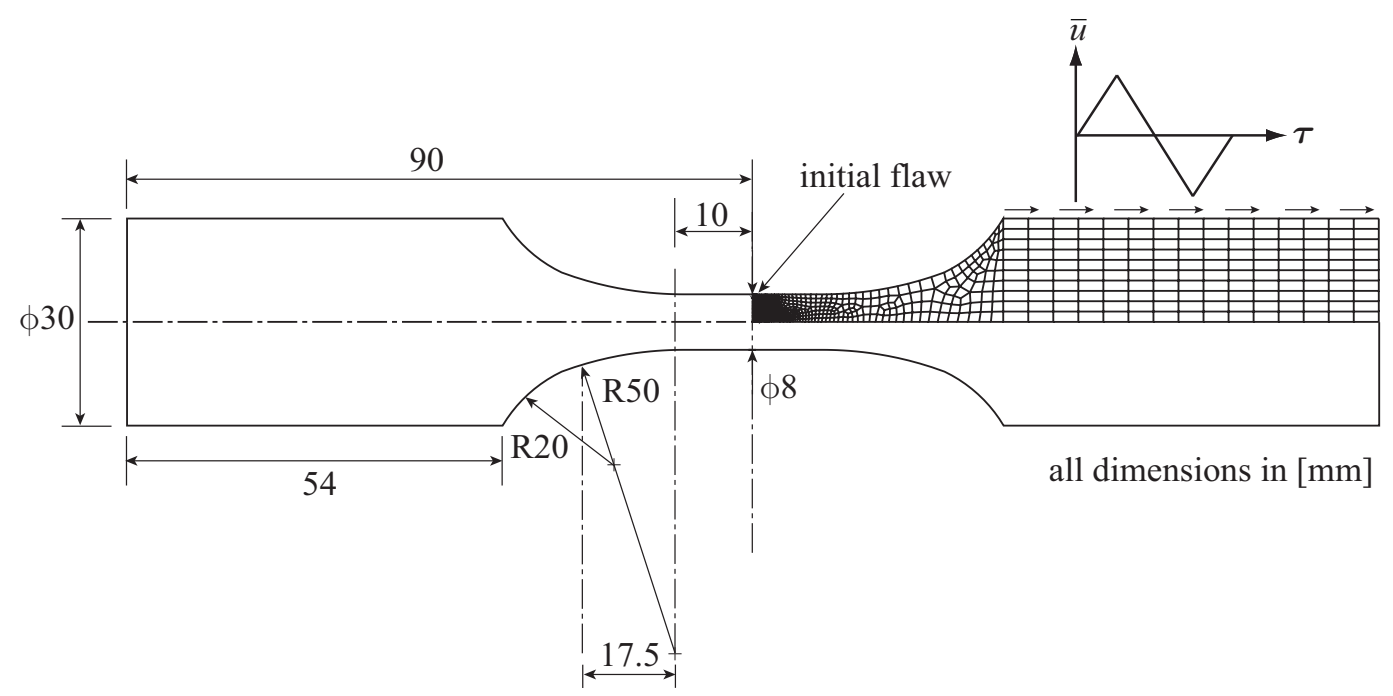

Figure 8: Geometry and discretization of the cylinderical fatigue test specimen made of 316L stainless steel.

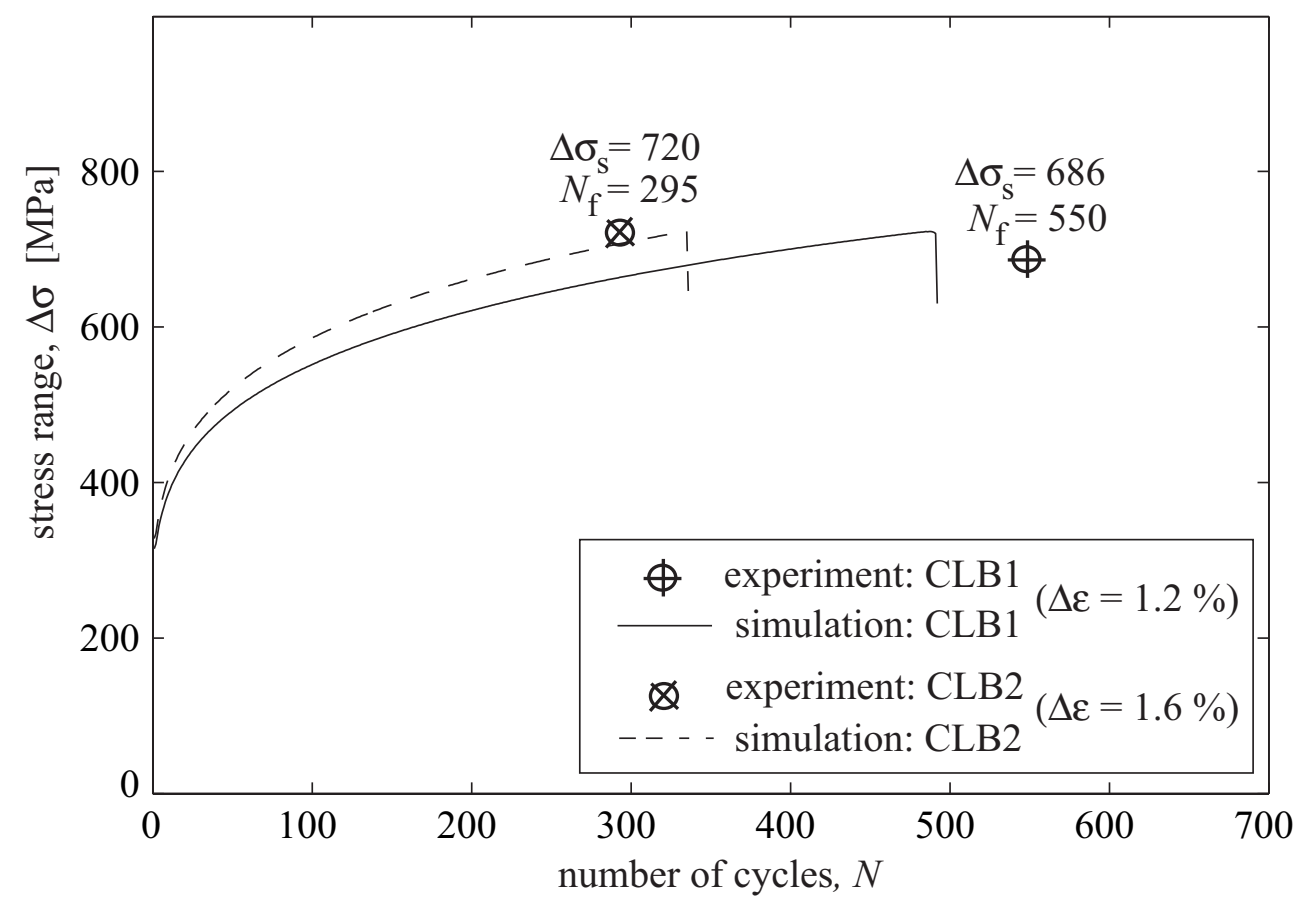

Figure 9: Variation of the stress range throughout simulations, CLB1 and CLB2, compared to experimental observations. 


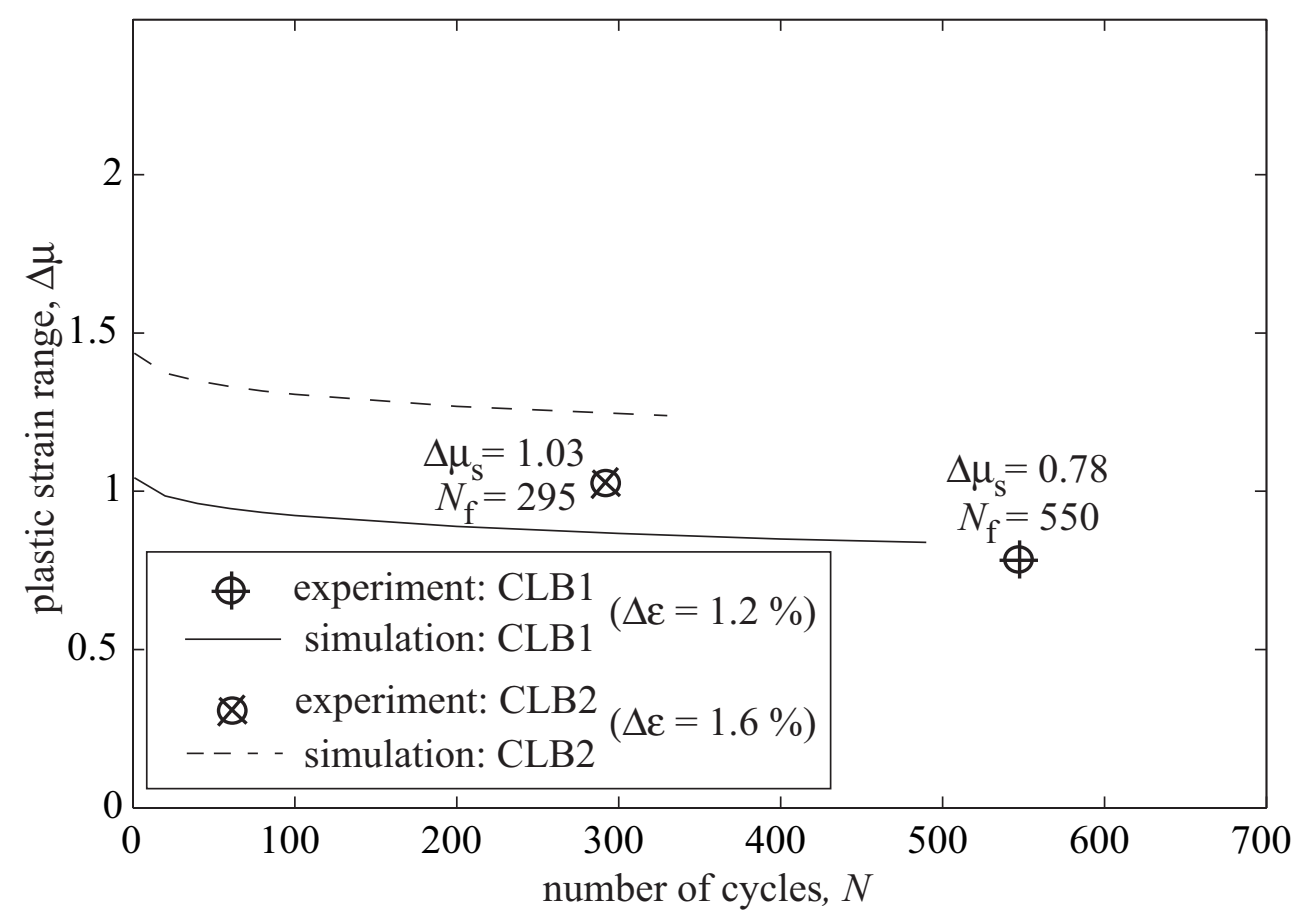

Figure 10: Variation of the plastic strain range throughout simulations, CLB1 and CLB2, compared to experimental observations.

of $5 \mathrm{kN}$ was applied to the pre-cracked specimen and the maximum amplitude of the loading was reduced to $3 \mathrm{kN}$ in the subsequent cycle. Growth of the cracks was observed during the loading period and crack growth curves are provided.

We conducted numerical simulations using the GTN model in the framework of the proposed multiscale life prediction methodology to predict the crack growth curves of the fatigue and overload specimens. Plane stress conditions were assumed and half of the geometry was modeled due to symmetry. The finite element mesh is illustrated in Fig. 11. The multiscale simulations were performed using the algorithm parameters, $E_{L}=4, \delta \boldsymbol{\omega}^{\text {all }}=\{0.05,0.05\}$, and with error tolerances of $\mathbf{E}^{t o l}=\{0.0075,0.0075\}$. The multiscale algorithm parameters are chosen to be relatively small to attain high levels of accuracy. The effects of the choice of algorithm parameters on the accuracy and performance of the model is presented in Ref. [34]. The initial void volume fraction is obtained using Franklin's empirical formula to be $f_{0}=0.012 \%$. The calibrated model parameters for fatigue life which are the Tvergaard constant, $q_{1}$, the mixed hardening parameter, $b$, and the strain hardening exponent, $n$, are set to the values identified in the calibration phase ( $q_{1}=1.1, b=0.22$, and $n=3.7$, respectively). The value of $0.2 \%$ proof stress is provided by Ref. [51] as $334 \mathrm{MPa}$.

Figure 13 illustrates the crack growth curves of the fatigue and overload specimens as predicted by the simulations along with the experimental observations. A reasonable overall match was observed between the experimental observations and the numerical predictions of both fatigue and overload specimens. The total life of the fatigue and overload specimens were 70000 and 130000 cycles, respectively. The proposed multiscale technique required the resolution of only 2530 and 2650 load cycles to evaluate the crack growth on the fatigue and overload specimens, respectively. 


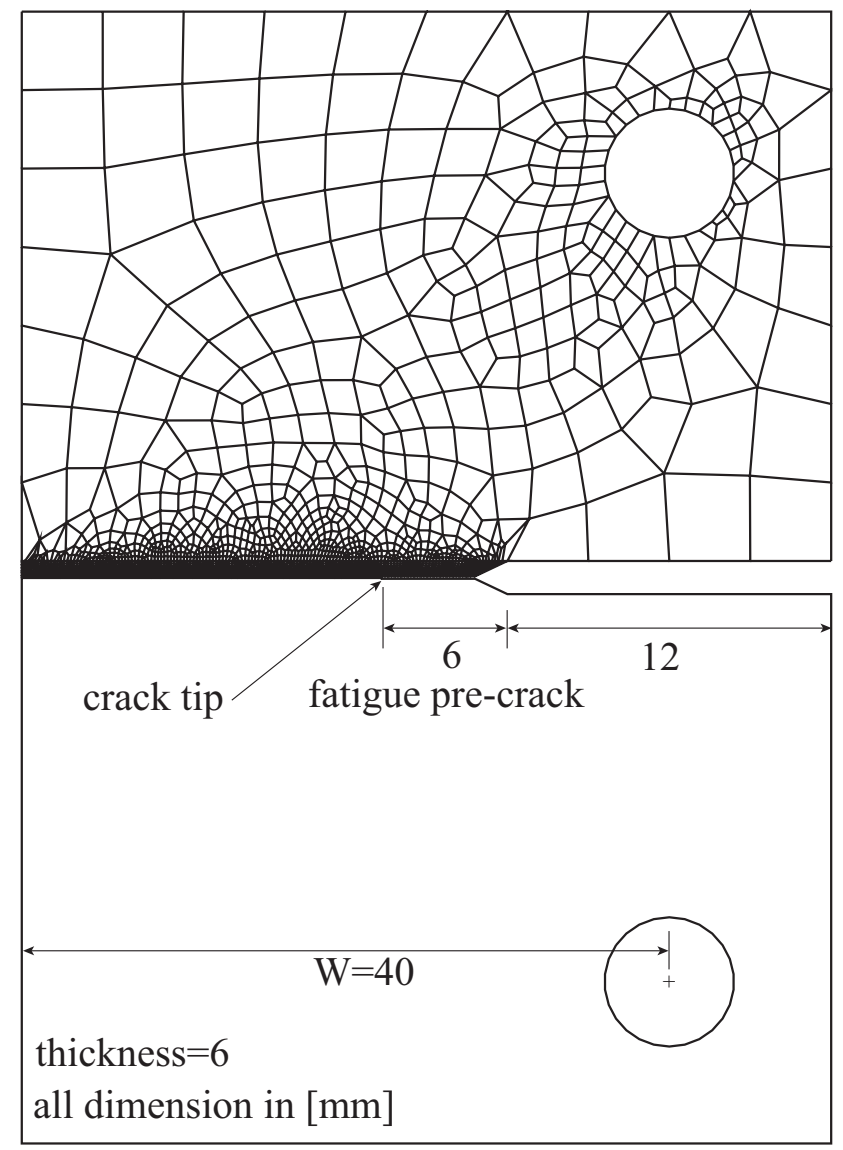

Figure 11: Geometry and discretization of the CT specimen made of 316L stainless steel. 


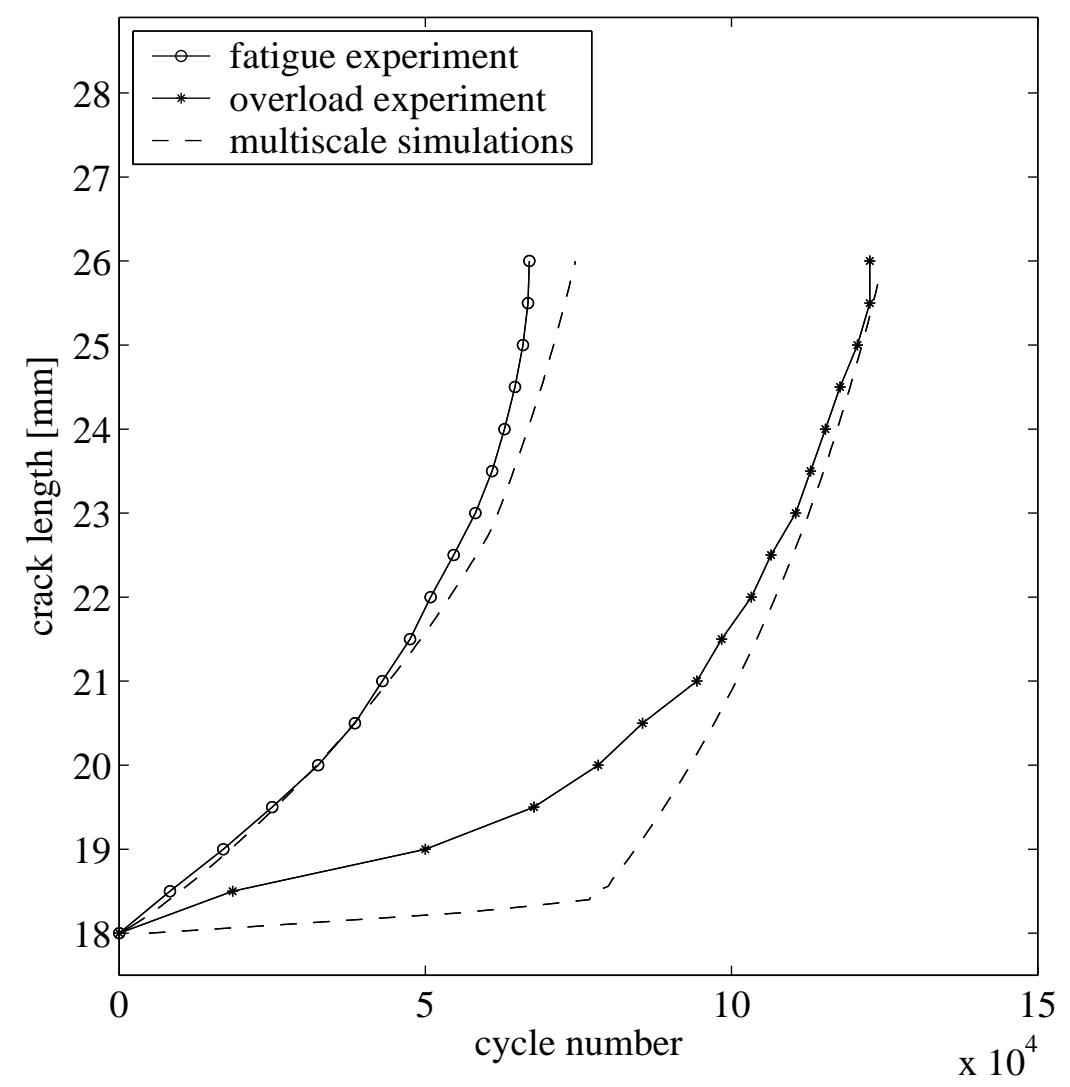

Figure 12: Experimental and predicted crack growth curves of the fatigue and overload specimens. 


\section{Discussion and conclusions}

The manuscript presents a computational fatigue life prediction methodology based on the mathematical homogenization theory with almost periodic fields. The almost-periodicity is a byproduct of irreversible processes, such as damage accumulation, which violates the condition of local periodicity. The nonlocal multiscale model has been shown to be insensitive to the mesh size as long as the characteristic size is smaller than the element size. Calibration studies revealed certain shortcomings of the model, particularly in the modeling of the stress-strain loops under high amplitude loading (very low cycle fatigue). Some of these deficiencies could be circumvented by employing multi-yield surfaces and/or bounding surface plasticity theories. Nevertheless, the calibrated nonlocal multiscale model performed reasonably well in validation studies more than justifying its usefulness due to significant computational cost savings (approximately 90-94\% cost reduction) compared to the direct cycle-by-cycle simulation.

\section{Acknowledgement}

This work was supported by the Office of Naval Research through grant number N00014-97-10687. This support is gratefully acknowledged.

\section{References}

[1] P. C. Paris and F. Erdogan. A critical analysis of crack propagation laws. J. Basic Engng., 85:528-534, 1963.

[2] R. G. Foreman, V. E. Keary, and R. M. Engle. Numerical analysis of crack propagation in cyclic-loaded structures. J. Basic Engng., 89:459-464, 1967.

[3] C. Gilbert, R. Dauskardt, and R. Ritchie. Microstructural mechanisms of cyclic fatigue-crack propagation in grain bridging ceramics. Ceramics International, 23:413-418, 1997.

[4] C. Laird. Mechanisms and Theories of Fatigue, page 149:203. American Society of Metals, 1972.

[5] O. E. Wheeler. Spectrum loading and crack growth. J. Basic Engng., 94:181-186, 1972.

[6] O. Nguyen, E. A. Repetto, M. Ortiz, and R. A. Radovitzky. A cohesive model of fatigue crack growth. Int. J. Fracture, 110(4):351-369, 2001.

[7] K. L. Roe and T. Siegmund. An irreversible cohesive zone model for interface fatigue crack growth simulation. Engng. Fracture Mech., 70:209-232, 2003.

[8] J. L. Chaboche. Continuum damage mechanics i: General concepts \& ii: Damage growth, crack initiation and crack growth. J. Appl. Mech., 55:59-72, 1988.

[9] C. L. Chow and Y. Wei. A model of continuum damage mechanics for fatigue failure. Int. J. Fatigue, 50:301-306, 1991. 
[10] J. Fish and Q. Yu. Computational mechanics of fatigue and life predictions for composite materials and structures. Comput. Meth. Appl. Mech. Engng., 191:4827-4849, 2002.

[11] M. Kaminski and M. Kleiber. Numerical homogenization of n-component composites including stochastic interface defects. Int. J. Numer. Meth. Engng., 47:1001-1027, 2000.

[12] G. W. Milton. The Theory of Composites. Cambridge Univ. Press, Cambridge, UK, 2002.

[13] S. Torquato. Random Heterogeneous Materials: Microstucture and Macroscopic Properties. Springer-Verlag, New York, 2002.

[14] K. Sab. On the homogenization and the simulation of random materials. Eur. J. Mech., A/Solids, 11:585-607, 1992.

[15] J. Fish and A. Wagiman. Multiscale finite element method for a locally nonperiodic heterogeneous medium. Comp. Mech., 12:164-180, 1993.

[16] S. Ghosh, K. Lee, and S. Moorthy. Multiple scale analysis of heterogeneous elastic structures using homogenization theory and voronoi cell finite element method. Int. J. Solids Structures, 32:27-62, 1995.

[17] S. Ghosh, K. Lee, and S. Moorthy. Two scale analysis of heterogeneous elastic-plastic materials with asymptotic homogenization and voronoi cell finite element method. Comput. Methods Appl. Mech. Engng., 132:63-116, 1996.

[18] J. T. Oden and T. I. Zohdi. Analysis and adaptive modeling of highly heterogeneous elastic structures. Comput. Methods. Appl. Mech. Engng., 148:367-391, 1997.

[19] T. I. Zohdi, J. T. Oden, and G. J. Rodin. Hierachical modeling of heterogeneous bodies. Comput. Meth. Appl. Mech. Engng., 138:273-298, 1996.

[20] N. Ansini and A. Braides. Separation of scales and almost-periodic effects in the asymptotic behavior of perforated media. Acta Applicandae Math., 65:59-81, 2001.

[21] A. Braides and A. Defranceschi. Homogenization of Multiple Integrals. Oxford Lecture Series in Mathematics and Its Applications. Oxford Univ. Press, Oxford, 1998.

[22] G. Nguetseng and H. Nnang. Homogenization of nonlinear monotone operators beyond the periodic setting. Electronic J. Diff. Eqn., 36:1-24, 2003.

[23] O. A. Oleinik, A. S. Shamaev, and G. A. Yosifian. Mathematical Problems in Elasticity and Homogenization. North-Holland Press, Amsterdam, 1992.

[24] V. V. Zhikov, S. M. Kozlov, and O. A. Oleinik. Homogenization of Differential Operators and Integral Functionals. Springer-Verlag, New York, 1994.

[25] V. Tvergaard. Material failure by void growth to coalescence. Adv. Appl. Mech., 27:83-151, 1990.

[26] R. de Borst and H. B. Muhlhaus. Gradient dependent plasticity: Formulation and algorithmic aspects. Int. J. Numer. Meth. Engng., 35:521-539, 1992. 
[27] Z. P. Bazant, T. B. Belytschko, and T. P. Chang. Continuum theory for strain softening. $J$. Engng. Mech., 110:1666-1691, 1984.

[28] R. de Borst and L. J. Sluys. Localization in cosserat continuum under static and dynamic loading conditions. Comput. Methods Appl. Mech. Engng., 90:805-827, 1991.

[29] K. Garikipati and T. J. R. Hughes. A variational multiscale approach to strain localization formulation for multidimensional problems. Comput. Methods. Appl. Mech. Engng., 188:3960, 2000.

[30] J. C. Simo and J. Oliver. A new approach to the analysis and simulation of strain softening in solids. In Z. P. Bazant, Z. Bittnar, M. Jirasek, and J. Mazars, editors, Fracture and Damage in Quasibrittle Structures. 1994.

[31] F. Zhou and J. F. Molinari. Dynamic crack propagation with cohesive elements: A methodology to address mesh dependency. Int. J. Numer. Meth. Engng., 59:1-29, 2004.

[32] R. H. J. Peerlings, W. A. M. Brekelmans, R. de Borst, and M. G. D. Geers. Gradient-enhanced damage modelling of high-cycle fatigue. Int. J. Numer. Meth. Engng., 49:1547-1569, 2000.

[33] C. Oskay and J. Fish. Fatigue life prediction using 2-scale temporal asymptotic homogenization. Int. J. Numer. Meth. Engng., 61:329-359, 2004.

[34] C. Oskay and J. Fish. Multiscale modeling of fatigue for ductile materials. Int. J. Comp. Multiscale Engng., 2, 2004.

[35] D. Cioranescu and P. Donato. An introduction to homogenization, volume 17 of Oxford lecture series in mathematics and its applications. Oxford Univ. Press, Oxford, UK, 1999.

[36] A. L. Gurson. Continuum theory of ductile rapture by void nucleation and growth: Part iyield criteria and flow rules for porous ductile media. J. Engng. Mater. and Technol., 99:2-15, 1977.

[37] C. C. Chu and A. Needleman. Void nucleation effects in biaxially stretched sheets. J. Engng. Mater. and Technol., 102:249-256, 1980.

[38] V. Tvergaard. Material failure by void coalescence in localized shear bands. Int. J. Solids Structures, 18:659-672, 1982.

[39] M. Gologanu, J.-B. Leblond, G. Perrin, and J. Devaux. Recent Extensions of Gurson's Model for Porous Ductile Metals, chapter 2, pages 61-130. Springer-Verlag, 1997.

[40] V. Tvergaard and A. Needleman. Analysis of the cup-cone fracture in a round tensile bar. Acta Metall., 32(1):157-169, 1984.

[41] J. Llorca, S. Suresh, and A. Needleman. An experimental and numerical study of cyclic deformation in metal-matrix composites. Metall. Trans. A, 23A:919-934, 1992.

[42] M. E. Mear and J. W. Hutchinson. Influence of yield surface curvature on flow localization in dilatant plasticity. Mech. Mater., 4:395-407, 1985. 
[43] G. Pijaudier-Cabot and Z. P. Bazant. Non-local damage theory. J. Eng. Mech., 113:1512$1533,1987$.

[44] V. Tvergaard and A. Needleman. Effects of nonlocal damage in porous plastic solids. Int. J. Solids Structures, 32:1063-1077, 1995.

[45] J. C. Simo. Strain softening and dissipation: A unification of approaches. In J. Mazars and Z. P. Bazant, editors, Cracking and Damage: Strain Localization and Size Effect, pages 440-461. Elsevier, New York, 1989.

[46] N. Aravas. On the numerical integration of a class of pressure-dependent plasticity models. Int. J. Numer. Meth. Engng., 24:1395-1416, 1987.

[47] J. H. Lee and Y. Zhang. On the numerical integration of a class of pressure-dependent plasticity models with mixed hardening. Int. J. Numer. Meth. Engng., 32:419-438, 1991.

[48] M. D. D. Geers, R. de Borst, W. A. M. Brekelmans, and R. H. J. Peerlings. Strain-based transient-gradient damage model for failure analyses. Comput. Methods. Appl. Mech. Engng., 160:133-153, 1998.

[49] H. J. Shi and G. Pluvinage. Cyclic stress-strain response during isothermal and thermomechanical fatigue. Int. J. Fatigue, 16:549-557, 1994.

[50] H. J. Shi, Z. G. Wang, and H. H. Su. Thermomechanical fatigue of a 3161 austenitic steel at two different temperature intervals. Scripta Materialia, 35:1107-1113, 1996.

[51] G. Wheatley, R. Niefanger, Y. Estrin, and X. Z. Hu. Fatigue crack growth in 3161 stainless steel. Key Engng. Matl., 145-149:631-636, 1998.

[52] V. Tvergaard. Effect of yield surface curvature and void nucleation on plastic flow localization. J. Mech. Phys. Solids, 35(1):43-60, 1987. 


\section{A Evaluation of the consistency parameter for the nonlocal GTN model}

The evaluation of the consistency parameter of the micro-chronological problem is similar to the computation of the consistency parameter of a single scale model (e.g., the boundary value problem defined in Eqs. (14) - (19)). To simplify the notation, we therefore present the evaluation of the consistency parameter using the response fields in total form. The consistency condition for the micro-chronological problem is defined as:

$$
\Phi_{, \tau}=0
$$

The local consistency parameter, $\lambda$, is evaluated based on the approach proposed in Ref [52]. To this extent, we define a fictitious yield surface, $\Phi^{G}$

$$
\Phi^{G}=\Phi^{G}\left(\boldsymbol{\sigma}^{G}, f, \sigma_{M}\right)=\frac{\left(q^{G}\right)^{2}}{\sigma_{M}^{2}}+2 \hat{f} \cosh \left(-\frac{3}{2} \frac{p^{G}}{\sigma_{M}}\right)-1-(\hat{f})^{2}
$$

The fictitious stress components, $\boldsymbol{\sigma}^{G}$, and the fictitious local rate of plastic strains chosen to be:

$$
\begin{aligned}
\frac{\boldsymbol{\sigma}^{G}}{\sigma_{M}} & =\frac{\mathbf{B}}{\boldsymbol{\sigma}_{F}} \\
\boldsymbol{\mu}_{, \tau} & =\boldsymbol{\mu}_{, \tau}^{G}
\end{aligned}
$$

Equation (68a) ensures that the yielding occurs simultaneously in $\Phi$ and $\Phi^{G}$ (i.e., $\Phi=0 \Rightarrow \Phi^{G}=0$ ). Equation (68b) states that the local rate of plastic strains is taken to be identical to that evaluated using the fictitious yield surface. Eq. (68b) must be satisfied at all material points in $\Omega$

$$
\bar{\lambda} \frac{\partial \Phi}{\partial \boldsymbol{\sigma}}=\bar{\lambda}^{G} \frac{\partial \Phi^{G}}{\partial \boldsymbol{\sigma}^{G}}
$$

The consistency condition (Eq. (66)) along with Eqs. (68) and (69) may be used to obtain an expression for the local consistency parameter in the form of an integral equation

$$
\lambda(\mathbf{x}, t)=\frac{\frac{\partial \phi}{\partial \boldsymbol{\sigma}}: \mathbf{L}: \boldsymbol{\varepsilon}_{, \tau}}{\frac{\partial \phi}{\partial \boldsymbol{\sigma}}: \mathbf{L}: \frac{\partial \phi}{\partial \boldsymbol{\sigma}}}-\frac{H}{\frac{\partial \phi}{\partial \boldsymbol{\sigma}}: \mathbf{L}: \frac{\partial \phi}{\partial \boldsymbol{\sigma}}} \frac{1}{W(\mathbf{x})} \int_{\Omega} w(\mathbf{x}, \mathbf{s}) \lambda(\mathbf{s}, t) d \mathbf{s}
$$

The plastic modulus, $H$, is given by:

$$
H=-\frac{\sigma_{M}}{\sigma_{F}}\left\{\frac{\partial \Phi}{\partial f}(1-f)\left(\boldsymbol{\delta}: \frac{\partial \Phi}{\partial \boldsymbol{\sigma}}\right)+\left[\frac{\sigma_{M}}{\sigma_{F}} \mathcal{A} \frac{\partial \Phi}{\partial f}+\frac{\partial \Phi}{\partial \sigma_{F}}\right] \frac{E^{t}}{1-f} \frac{1}{\sigma_{M}}\left(\boldsymbol{\Theta}: \frac{\partial \Phi}{\partial \boldsymbol{\sigma}}\right)\right\}
$$




\section{B Evolution equations for the nonlocal GTN model}

The evolution equations of the internal state variables of the GTN model (i.e., void volume fraction, equivalent plastic strain, matrix flow strength, and the center of the yield surface) for micro- and macro-chronological problems is derived herein.

To simplify the presentation, we define:

$$
\begin{aligned}
\overline{\boldsymbol{\mu}}_{, \tau} & =\bar{\lambda}^{1} \frac{\partial \Phi}{\partial \boldsymbol{\sigma}} \\
\mathfrak{M}(\overline{\boldsymbol{\mu}})_{, t} & =\bar{\lambda}^{\mathrm{o}}\left\langle\frac{\partial \Phi}{\partial \boldsymbol{\sigma}}\right\rangle+\frac{1}{\zeta}\left\langle\overline{\boldsymbol{\mu}}_{, \tau}\right\rangle
\end{aligned}
$$

Decomposing the void volume fraction using Eq. (35), and introducing the resulting fields as well as Eqs. (72) into the original evolution equation of the void volume fraction

$$
\begin{aligned}
O\left(\zeta^{-1}\right): & \tilde{f}_{, \tau} & =q_{1}(1-\mathfrak{M}(f)-\tilde{f}) \boldsymbol{\delta}: \overline{\boldsymbol{\mu}}_{, \tau}+\mathcal{A} \tilde{\rho}_{, \tau} \\
O(1): & \mathfrak{M}(f)_{, t}+\tilde{f}_{, t} & =q_{1}(1-\mathfrak{M}(f)-\tilde{f}) \boldsymbol{\delta}:\left(\mathfrak{M}(\overline{\boldsymbol{\mu}})_{, t}+\overline{\boldsymbol{\mu}}_{, t}\right)+\mathcal{A}\left(\mathfrak{M}(\rho)_{, t}+\tilde{\rho}_{, t}\right)(7)
\end{aligned}
$$

The micro-chronological evolution equation for the void volume fraction is given by (73). Applying the PTH operator to Eqs. (73) and (74) and using Eq. (36) yields:

$$
\begin{array}{r}
\mathfrak{M}(f)_{, t}=q_{1}(1-\mathfrak{M}(f)-\langle\tilde{f}\rangle) \boldsymbol{\delta}: \mathfrak{M}(\overline{\boldsymbol{\mu}})_{, t}-q_{1}\left\langle\tilde{f} \boldsymbol{\delta}:\left(\frac{1}{\zeta} \overline{\boldsymbol{\mu}}_{, \tau}+\overline{\boldsymbol{\mu}}_{, t}\right)\right\rangle \\
+\langle\mathcal{A}\rangle \mathfrak{M}(\rho)_{, t}-\left\langle\mathcal{A}\left(\frac{1}{\zeta} \tilde{\boldsymbol{\rho}}_{, \tau}+\tilde{\boldsymbol{\rho}}_{, t}\right)\right\rangle
\end{array}
$$

which is the evolution equation of the macro-chronological void volume fraction.

The evolution equations of the micro-chronological and macro-chronological equivalent plastic strains may be derived using a similar algebra

$$
\begin{gathered}
\tilde{\rho}_{, \tau}=\frac{(\mathfrak{M}(\mathbf{B})+\tilde{\mathbf{B}}): \overline{\boldsymbol{\mu}}_{, \tau}}{[1-\mathfrak{M}(f)-\tilde{f}]\left[\mathfrak{M}\left(\sigma_{F}\right)+\tilde{\boldsymbol{\sigma}}_{F}\right]} \\
\mathfrak{M}(\rho)_{, t}=\left\langle\frac{(\mathfrak{M}(\mathbf{B})+\tilde{\mathbf{B}})}{[1-\mathfrak{M}(f)-\tilde{f}]\left[\mathfrak{M}\left(\sigma_{F}\right)+\tilde{\sigma}_{F}\right]}\right\rangle: \mathfrak{M}(\overline{\boldsymbol{\mu}})_{, t} \\
+\left\langle\frac{(\mathfrak{M}(\mathbf{B})+\tilde{\mathbf{B}})}{[1-\mathfrak{M}(f)-\tilde{f}]\left[\mathfrak{M}\left(\sigma_{F}\right)+\tilde{\boldsymbol{\sigma}}_{F}\right]}:\left(\frac{1}{\zeta} \overline{\boldsymbol{\mu}}_{, \tau}+\overline{\boldsymbol{\mu}}_{, t}\right)\right\rangle
\end{gathered}
$$

The evolution equations of the micro- and macro-chronological matrix flow strength and the radius of the yield surface may be expressed as:

$$
\begin{aligned}
\tilde{\sigma}_{M, \tau} & =E^{t} \tilde{\rho}_{, \tau} \\
\mathfrak{M}\left(\sigma_{M}\right)_{, t} & =\left\langle E^{t}\right\rangle \mathfrak{M}(\rho)_{, t}+\left\langle E^{t}\left(\frac{1}{\zeta} \tilde{\rho}_{, \tau}+\tilde{\rho}_{, t}\right)\right\rangle
\end{aligned}
$$


and,

$$
\begin{aligned}
\tilde{\sigma}_{F, \tau} & =b \tilde{\sigma}_{M, \tau} \\
\mathfrak{M}\left(\sigma_{F}\right)_{, t} & =b \mathfrak{M}\left(\sigma_{M}\right)_{, t}
\end{aligned}
$$

in which, Eqs. (78) and (79) are the micro- and macro-chronological evolution equations of the matrix flow strength, respectively. Equations (80) and (81) correspond to the evolution equations of the yield surface radius for the micro- and macro-chronological problems, respectively.

Decomposing the yield surface center, $\boldsymbol{\alpha}$, according to Eq. (35), and introducing the micro- and macro-chronological consistency parameters to Eq. (34)

$$
\begin{aligned}
O\left(\zeta^{-1}\right): & \tilde{\boldsymbol{\alpha}}_{, \tau} & =\bar{\lambda}^{1} Q(\mathfrak{M}(\mathbf{B})+\tilde{\mathbf{B}}) \\
O(1): & \mathfrak{M}(\boldsymbol{\alpha})_{, t}+\tilde{\boldsymbol{\alpha}}_{, t} & =\bar{\lambda}^{\mathrm{o}} Q(\mathfrak{M}(\mathbf{B})+\tilde{\mathbf{B}})
\end{aligned}
$$

in which,

$$
Q=(1-b)\left(\mathbf{B}: \frac{\partial \Phi}{\partial \boldsymbol{\sigma}}\right)^{-1}\left[H+\frac{\sigma_{y}}{\sigma_{F}} \frac{\partial \Phi}{\partial f}\left\{q_{1}(1-f)\left(\boldsymbol{\delta}: \frac{\partial \Phi}{\partial \boldsymbol{\sigma}}\right)+\frac{E^{t}}{1-f} \frac{1}{\sigma_{F}}\left(\mathbf{B}: \frac{\partial \Phi}{\partial \boldsymbol{\sigma}}\right)\right\}\right]
$$

The micro-chronological evolution equation of the yield surface center is given as Eq. (82). Applying the PTH operator to Eqs. (82) and (83) yields the macro-chronological evolution equation of the yield surface center

$$
\mathfrak{M}(\boldsymbol{\alpha})_{, t}=\bar{\lambda}^{o}(\langle Q\rangle \mathfrak{M}(\mathbf{B})+\langle Q \tilde{\mathbf{B}}\rangle)+\frac{1}{\zeta}\left\langle\tilde{\boldsymbol{\alpha}}_{, \tau}\right\rangle
$$

